\title{
THE EXISTENCE OF SOLITON METRICS FOR NILPOTENT LIE GROUPS
}

\author{
TRACY L. PAYNE
}

\begin{abstract}
We show that a left-invariant metric $g$ on a nilpotent Lie group $N$ is a soliton metric if and only if a matrix $U$ and vector $v$ associated the manifold $(N, g)$ satisfy the matrix equation $U v=[1]$, where [1] is a vector with every entry a one. We associate a generalized Cartan matrix to the matrix $U$ and use the theory of Kac-Moody algebras to analyze the solution spaces for such linear systems. We use these methods to find infinitely many new examples of nilmanifolds with soliton metrics. We give a sufficient condition for a sum of soliton metric nilpotent Lie algebra structures to be soliton, and we use this criterion to show that a soliton metric exists on every naturally graded filiform metric Lie algebra.
\end{abstract}

\section{INTRODUCTION}

1.1. Overview. A nilsoliton metric is a left-invariant metric on a nilpotent Lie group such that the Ricci endomorphism, viewed as a linear mapping on the Lie algebra, differs from a scalar multiple of the identity map by a derivation. This condition may be viewed as the Einstein condition at the second level of Lie algebra cohomology (relative to the adjoint representation).

Nilsoliton metrics are of interest for several reasons. First, nonabelian nilpotent Lie groups do not admit left-invariant Einstein metrics, and nilsoliton metrics have nice properties that make them preferred metrics on nilpotent Lie groups in the absence of Einstein metrics. J. Lauret has shown that given a nilpotent Lie group, a nilsoliton metric is unique up to scaling, and that nilsoliton metrics arise as critical points of a natural Riemannian functional on the space of normalized nilpotent Lie algebra brackets ([Lau01a $]$ ). Special metrics on nilpotent Lie groups, such as metrics from horospheres of symmetric spaces of noncompact type, and more generally, groups of type $H$, are

2000 Mathematics Subject Classification. Primary: 53C25; Secondary: 53C30, 22E25, 22F 30 .

Key words and phrases. homogeneous space - nilmanifold - nilsoliton metric soliton metric - weighted Dynkin diagram . 
nilsoliton metrics. However, nilsoliton metrics fail to have the desirable property of existence: there exist many nilpotent Lie algebras that do not admit nontrivial semisimple derivations and hence the corresponding nilpotent groups do not admit nilsoliton metrics.

Second, soliton metrics arise in the study of Ricci flow, which was introduced by Hamilton in Ham82 as part of his program to prove Thurston's Geometrization Conjecture for compact three-manifolds. Perelman has recently made great progress ([Pera, [Perc], Perb]) toward the completion of that program. Einstein metrics are the fixed points for the normalized Ricci flow on the space of Riemannian metrics on a compact manifold. A solution to the Ricci flow which moves by diffeomorphisms and also dilates by a time-dependent factor is called a Ricci soliton. Ricci soliton metrics may be viewed as generalized fixed points for the Ricci flow. A left-invariant metric on a nilpotent Lie group is a Ricci soliton metric if and only if it is a nilsoliton metric (Prop. 1.1, [Lau01a]).

Third, nilsoliton metrics are inherent in the study of Einstein metrics on noncompact homogeneous spaces. All known noncompact, nonflat, homogeneous Einstein manifolds are isometric to solvable Lie groups endowed with left-invariant metrics (solvmanifolds). D. V. Alekse'evskii conjectured that an isotropy subgroup of the isometry group of a noncompact, nonflat, homogeneous Einstein manifold is a maximal compact subgroup. If Alekse'evskii's conjecture is true, then any noncompact, nonflat, homogeneous Einstein manifold with linear isometry group would be isometric to a solvmanifold.

It has been shown that left-invariant Einstein metrics on unimodular solvable groups are flat ([DM82]). The remaining case of interest is when the solvable group is nonunimodular. In [Heb98], J. Heber gave a comprehensive analysis of structure of nonunimodular Einstein solvmanifolds and their moduli spaces. We say that a metric Lie algebra is Einstein (or nilsoliton) if the corresponding simply connected homogeneous space is Einstein (or nilsoliton). A metric solvable Lie algebra $\mathfrak{s}$ is called standard if $\mathfrak{a}:=[\mathfrak{s}, \mathfrak{s}]^{\perp}$ is abelian. All Einstein solvable metric Lie algebras are of standard type ([Lau $]$ ). Heber showed that any nonunimodular solvable Lie algebra $\mathfrak{s}$ endowed with a standard Einstein metric $Q$ has a subalgebra $\mathfrak{s}_{0}:=\langle H\rangle \oplus \mathfrak{n}$ so that $\left(\mathfrak{s}_{0},\left.Q\right|_{\mathfrak{s}_{0}}\right)$ is Einstein. Thus, the analysis of standard nonunimodular Einstein solvmanifolds is reduced to the case where $\mathfrak{a}$ has dimension one (the rank one case).

In [Lau01a] and [Lau01b], building on the work of Heber, J. Lauret established a correspondence between rank one Einstein metric solvable Lie algebras and nilsoliton metric Lie algebras. A metric solvable 
extension of a metric nilpotent Lie algebra $(\mathfrak{n}, Q)$ with Lie bracket $[\cdot, \cdot]_{\mathfrak{n}}$ is a solvable Lie algebra $\mathfrak{s}=\mathfrak{a} \oplus \mathfrak{n}$ with Lie bracket $[\cdot, \cdot]_{\mathfrak{s}}$ and inner product $\tilde{Q}$ such that $[\mathfrak{s}, \mathfrak{s}]_{\mathfrak{s}}=\mathfrak{n}=\mathfrak{a}^{\perp},\left.[\cdot, \cdot]_{\mathfrak{s}}\right|_{\mathfrak{n}}=[\cdot, \cdot]_{\mathfrak{n}}$, and $\left.\tilde{Q}\right|_{\mathfrak{n} \times \mathfrak{n}}=Q$. Lauret proved that a metric nilpotent Lie algebra $(\mathfrak{n}, Q)$ is nilsoliton if and only if $(\mathfrak{n}, Q)$ admits a metric solvable extension $(\mathfrak{s}=\mathfrak{a} \oplus \mathfrak{n}, \tilde{Q})$ such that $\mathfrak{a}$ is abelian and the corresponding solvmanifold $(S, \tilde{g})$ is Einstein. (Theorem 3.7 of [Lau01b]). This theorem may be used to translate theorems in this paper about nilsoliton metrics to parallel statements about Einstein solvmanifolds. Given a nilsoliton metric Lie algebra, it is the semisimple derivation $D$, the difference of the Ricci endomorphism and the scalar multiple of the identity map, that defines the Einstein solvable extension: that is, $\mathfrak{s}:=\mathbb{R} H \oplus \mathfrak{n}$ and $[\cdot, \cdot]_{\mathfrak{n}}$ is extended to $[\cdot, \cdot]_{\mathfrak{s}}$ by setting $[H, X]_{\mathfrak{s}}:=D X$ for $X \in \mathfrak{n}$. Nonabelian nilpotent Lie algebras do not admit Einstein metrics ([Jen69, see also Theorem [6), so the derivation $D$ for a nilsoliton metric Lie algebra is trivial if and only if $\mathfrak{n}$ is abelian.

In this work, we study nilsoliton metrics using algebraic and combinatorial methods. We work in the class of metric (nonassociative skew) algebras. A metric algebra $\left(\mathfrak{n}_{\mu}, Q\right)$ is a vector space $\mathfrak{n}$ equipped with an inner product $Q$ and an algebraic structure defined by an element $\mu: \mathfrak{n} \times \mathfrak{n} \rightarrow \mathfrak{n}$ in the vector space $\Lambda^{2} \mathfrak{n}^{*} \otimes \mathfrak{n}$ of skew-symmetric bilinear vector-valued maps. The expression for the Ricci form for a nilmanifold extends to define a "nil-Ricci" endomorphism $\mathrm{Ric}_{\mu}$ for each metric algebra. Our main interest is in nilpotent algebras. If $\operatorname{Ric}_{\mu}-\beta \mathrm{Id}$ is a derivation of $\mathfrak{n}_{\mu}$ we say that $\left(\mathfrak{n}_{\mu}, Q\right)$ satisfies the nilsoliton condition with nilsoliton constant $\beta$.

We associate to each nonabelian metric algebra $\left(\mathfrak{n}_{\mu}, Q\right)$ and orthonormal basis $\mathcal{B}$ of eigenvectors for the nil-Ricci endomorphism an array of combinatorial objects: a set $\Lambda$ of integer triples encoding the nontrivial structure constants for the basis, a set of vectors with entries of zero, one and minus one indexed by $\Lambda$, a Gram matrix $U$ encoding the inner products of these vectors, and graph $S(U)$ defined by the matrix $U$. We will see that interesting algebraic and geometric properties of $\left(\mathfrak{n}_{\mu}, Q\right)$ and $\mathcal{B}$ are displayed in properties of these combinatorial objects.

We associate to $\left(\mathfrak{n}_{\mu}, Q\right)$ a vector $\left[\alpha^{2}\right]$ listing the squares of the nontrivial structure constants. It is shown that the nil-Ricci endomorphism satisfies the nilsoliton condition if and only if the vector $\left[\alpha^{2}\right]$ is a solution to the matrix equation $U v=[1]$ where [1] is a column vector with every entry a one, thus reducing the difficult tensorial problem of finding Einstein and soliton metrics to a problem of linear algebra. 
We define a generalized Cartan matrix in terms of the Gram matrix $U$ and apply results from the theory of Kac-Moody algebras to analyze the existence, uniqueness, and properties of solutions to the special systems $U v=[1]$. From these results we get a procedure for finding all nilsoliton metrics in a fixed dimension, and hence all rank one Einstein solvmanifolds of any given dimension. Once we have a rank one Einstein solvmanifold it is not hard to find all higher rank extensions by computing the derivation algebra of the nilradical.

We study the linear systems arising from some examples, including nilpotent Lie groups associated to rank one symmetric spaces of noncompact type, and families of filiform nilpotent Lie groups.

When elements $\mu_{1}$ and $\mu_{2}$ in $\Lambda^{2} \mathfrak{n}^{*} \otimes \mathfrak{n}$ define algebras $\mathfrak{n}_{\mu_{1}}$ and $\mathfrak{n}_{\mu_{2}}$ on the inner product space $(\mathfrak{n}, Q)$ so that the metric algebras $\left(\mathfrak{n}_{\mu_{1}}, Q\right)$ and $\left(\mathfrak{n}_{\mu_{2}}, Q\right)$ satisfy the nilsoliton condition, in general the sum $\mu_{1}+\mu_{2}$ does not define an algebra $\mathfrak{n}_{\mu_{1}+\mu_{2}}$ so that $\left(\mathfrak{n}_{\mu_{1}+\mu_{2}}, Q\right)$ again satisfies the nilsoliton condition; a sufficient condition to guarantee this is found. This criterion is useful for constructing nilsoliton metrics, and for showing in certain situations that a metric Lie algebra of interest is nilsoliton.

1.2. Summary of Results. A nilmanifold is a connected Riemannian manifold with a transitive nilpotent group of isometries. A nilmanifold is isometric to a nilpotent Lie group $N$ endowed with a left-invariant Riemannian metric $g$. P. Eberlein and others have studied the geometry of two-step nilmanifolds in depth (see Ebe04 for a survey and references), but little has been done in the general higher-step case, where the expressions for the Levi-Civita connection and the exponential map are markedly more complicated than in the two-step case.

We associate to the nilmanifold $(N, g)$ the metric Lie algebra $(\mathfrak{n}, Q)$, where $\mathfrak{n}$ is the Lie algebra of $N$ and $Q$ is the restriction of the metric $g$ on $N$ to the tangent space at the identity $T_{e} N \cong \mathfrak{n}$. As we are always interested in purely local properties, we identify $(N, g)$ with $(\mathfrak{n}, Q)$, and when we refer to things like the connection, curvature, etc. for the metric Lie algebra $(\mathfrak{n}, Q)$, we mean the structures associated to $(\mathfrak{n}, Q)$ coming from the connection, curvature, etc. for the homogeneous space $(N, g)$.

Although our primary aim is to investigate nilsoliton metrics for metric nilpotent Lie algebras, it is convenient to generalize the setting and allow $\left(\mathfrak{n}_{\mu}, Q\right)$ to be a metric algebra, where $\mu=[\cdot, \cdot]$ is in $\Lambda^{2} \mathfrak{n}^{*} \otimes \mathfrak{n}$. Let $\mathcal{B}=\left\{X_{i}\right\}_{i=1}^{n}$ be a $Q$-orthonormal basis of $\mathfrak{n}_{\mu}$. (We always assume that bases are ordered.) The nil-Ricci endomorphism $\mathrm{Ric}_{\mu}$ is defined 
as $\left\langle\operatorname{Ric}_{\mu} X, Y\right\rangle=\operatorname{ric}_{\mu}(X, Y)$, where

(1)

$\operatorname{ric}_{\mu}(X, Y)=-\frac{1}{2} \sum_{i=1}^{n}\left\langle\left[X, X_{i}\right],\left[Y, X_{i}\right]\right\rangle+\frac{1}{4} \sum_{i, j=1}^{n}\left\langle\left[X_{i}, X_{j}\right], X\right\rangle\left\langle\left[X_{i}, X_{j}\right], Y\right\rangle$

for $X, Y \in \mathfrak{n}_{\mu}$. (We often write an inner product $Q(\cdot, \cdot)$ as $\langle\cdot, \cdot\rangle$.) When $\mathfrak{n}$ is a nilpotent metric Lie algebra, the nil-Ricci endomorphism is the Ricci endomorphism. If all elements of the basis are eigenvectors for the nil-Ricci endomorphism Ric $_{\mu}$, we call the orthonormal basis a Ricci eigenvector basis. By the symmetry of the nil-Ricci form, a Ricci eigenvector basis always exists.

Now we define some combinatorial objects associated to a set of integer triples $\Lambda \subset\{(i, j, k) \mid 1 \leq i, j, k \leq n\}$. We will later consider subsets of the following sets:

$$
\begin{aligned}
& \Upsilon_{n}=\{(i, j, k) \mid 1 \leq i<j \leq n, 1 \leq k \leq n\} \\
& \Psi_{n}=\{(i, j, k) \mid 1 \leq i<j \leq n, 1 \leq k \leq n, i \neq k, j \neq k\} \\
& \left.\Theta_{n}=\{(i, j, k)\} \mid 1 \leq i<j<k \leq n\right\}
\end{aligned}
$$

For $1 \leq i, j, k \leq n$, define the $1 \times n$ row vector $Y_{i j}^{k}$ to be $\varepsilon_{i}^{T}+\varepsilon_{j}^{T}-$ $\varepsilon_{k}^{T}$, where $\left\{\varepsilon_{i}\right\}_{i=1}^{n}$ is the standard orthonormal basis for $\mathbb{R}^{n}$. We call the vectors in $\left\{Y_{i j}^{k} \mid(i, j, k) \in \Lambda\right\}$ root vectors for $\Lambda$. Let $Y_{1}, Y_{2}, \ldots, Y_{m}$ (where $m=\|\Lambda\|$ ) be an enumeration of the root vectors in dictionary order. We define the root matrix $Y_{\Lambda}$ for $\Lambda$ to be the $m \times n$ matrix whose rows are the root vectors $Y_{1}, Y_{2}, \cdots, Y_{m}$. The Gram matrix $U_{\Lambda}$ for $\Lambda$ is the $m \times m$ matrix defined by $U_{\Lambda}=Y_{\Lambda} Y_{\Lambda}^{T}$; the $(i, j)$ entry of $U_{\Lambda}$ is the inner product of the $i$ th and $j$ th root vectors. Because there are a finite number of root vectors in any dimension, there are only finitely many possible root matrices and Gram matrices in any dimension.

The Gram matrix $U=\left(u_{i j}\right)$ defines a graph $S(U)$, which we will call the generalized Dynkin diagram of $U$. The vertices of the generalized Dynkin diagram are in one-to-one correspondence with triples $(i, j, k)$ in $\Lambda$. Let these vertices be named $n_{1}, n_{2}, \ldots n_{m}$ when listed in dictionary order. Between vertices $n_{i}$ and $n_{j}$ where $i \neq j$ and $u_{i j} \neq 0$, draw an edge and label it with $u_{i j}$. If the label is one, the label is omitted and if the label is minus one, the edge may be drawn as a dashed line segment. If $u_{i j}=0$, the vertices $n_{i}$ and $n_{j}$ are not connected by an edge. Technically, $S(U)$ is not a generalized Dynkin diagram as defined in [Kac90] since $U$ is not a generalized Cartan matrix. There is a true generalized Cartan matrix $A$ associated to $U$ when $\Lambda \subset \Psi_{n}$. Its edgeweighted graph $S(A)$ is closely related to the graph $S(U)$; however, we work work with $S(U)$ as $U$ is more natural than $A$ from a geometric 
point of view, and $U$ is uniquely defined, whereas there are different good choices for the matrix $A$ depending on the situation.

Next we define some structures associated to a nonabelian $n$-dimensional metric algebra $\left(\mathfrak{n}_{\mu}, Q\right)$ and an orthonormal basis $\mathcal{B}$ for $\left(\mathfrak{n}_{\mu}, Q\right)$. Let $\alpha_{i j}^{k}$ denote the structure constant $\left\langle\left[X_{i}, X_{j}\right], X_{k}\right\rangle$. The set $\Lambda\left(\mathfrak{n}_{\mu}, \mathcal{B}\right)=$ $\left\{(i, j, k) \mid i<j, \alpha_{i j}^{k} \neq 0\right\}$ indexes the set of nonzero structure constants, ignoring repetitions due to skew-symmetry. The set of triples $\Lambda\left(\mathfrak{n}_{\mu}, \mathcal{B}\right)$ is a subset of $\Upsilon_{n}$. We may say that the root vectors, root matrix, and Gram matrix for $\Lambda=\Lambda\left(\mathfrak{n}_{\mu}, Q\right)$ are for $\left(\mathfrak{n}_{\mu}, Q\right)$ with respect to the basis $\mathcal{B}$. Let $\left[\alpha^{2}\right]_{\left(\mathfrak{n}_{\mu}, \mathcal{B}\right)}$ denote the $m \times 1$ vector with the entries $\left(\alpha_{i j}^{k}\right)^{2}$ for $(i, j, k) \in \Lambda\left(\mathfrak{n}_{\mu}, \mathcal{B}\right)$, again taken in dictionary order. We call $\left[\alpha^{2}\right]_{\left(\mathfrak{n}_{\mu}, \mathcal{B}\right)}$ the structure vector for $\left(\mathfrak{n}_{\mu}, Q\right)$ with respect to $\mathcal{B}$. The structure vector $\left[\alpha^{2}\right]$ defines a weighting $w$ of the vertices of $S(U)$, assigning the $i$ th vertex $n_{i}$ the $i$ th entry of $\left[\alpha^{2}\right]$, so that the vertex for the triple $(i, j, k)$ has the weight $\left(\alpha_{i j}^{k}\right)^{2}$. We will sometimes abbreviate our notation, for example writing $\Lambda$ for $\Lambda\left(\mathfrak{n}_{\mu}, \mathcal{B}\right)$ or omitting subscripts, when it is clear what is meant. A single nilmanifold may have different root vectors, structure vectors, and Gram matrices, depending on the choice of Ricci eigenvector basis. Nonisometric nilmanifolds may have the same Gram matrices, structure vectors, and Dynkin diagrams.

In Section 3, we give simple formulas for computing Ricci and sectional curvature for metric Lie algebras relative to Ricci eigenvector bases, and we illustrate these formulas with examples.

In Section 4, we show that a metric algebra $\left(\mathfrak{n}_{\mu}, Q\right)$ satisfies the nilsoliton condition if and only if the structure vector with respect to $\left(\mathfrak{n}_{\mu}, Q\right)$ and any Ricci eigenvector basis $\mathcal{B}$ is a solution to a linear system associated to $\left(\mathfrak{n}_{\mu}, Q\right)$. We use $[a]_{m \times n}$ to denote the $m \times n$ matrix all of whose entries are the real number $a$.

Theorem 1. Let $\left(\mathfrak{n}_{\mu}, Q\right)$ be a nonabelian metric algebra with Ricci eigenvector basis $\mathcal{B}$. Let $U$ and $\left[\alpha^{2}\right]$ be the Gram matrix and the structure vector for $\left(\mathfrak{n}_{\mu}, Q\right)$ with respect to $\mathcal{B}$. Then $\left(\mathfrak{n}_{\mu}, Q\right)$ satisfies the nilsoliton condition with nilsoliton constant $\beta$ if and only if $U\left[\alpha^{2}\right]=$ $-2 \beta[1]_{m \times 1}$.

Thus, to find all nilsoliton metrics, one needs to find all vectors $v$ with positive entries that are solutions to systems of the form $U v=$ $-2 \beta[1]_{m \times 1}$, where $U$ is the Gram matrix defined by a set of root vectors. Choosing structure constants whose squares are the entries of $v$ will define a metric algebra $\left(\mathfrak{n}_{\mu}, Q\right)$ satisfying the nilsoliton condition. The sets of integer triples are enumerable, so this gives an algorithm for finding nilsolitons. 
A solution to the equation $U v=-2 \beta[1]_{m \times 1}$ gives a weighting $w$ on the vertices of the Dynkin diagram $S(U)$ with the property that for all $i$,

$$
3 w\left(n_{i}\right)+\sum_{u_{i j} \neq 0} u_{i j} w\left(n_{j}\right)=-2 \beta
$$

that is, thrice the value of the weighting at a vertex $n_{i}$ plus the edgeweighted sum of the values at adjacent vertices is constant. This interpretation of solutions allows one to use the symmetries of the generalized Dynkin diagram $S(U)$ to find symmetries in the solution space of the system $U v=-2 \beta[1]_{m \times 1}$.

Elementary properties of the Gram matrices for metric nilpotent Lie algebras are derived in Section 4. In Section 5, Theorem 13 interprets the Jacobi condition for a metric nilpotent algebra $\mathfrak{n}$ in terms of the weighted graph $S\left(U_{\Lambda}\right)$, so that one may check whether $\mathfrak{n}$ is a Lie algebra by looking at the graph $S\left(U_{\Lambda}\right)$. The proof of Theorem 1 is in Section [6, along with some theorems describing properties of derivations and gradings of nilpotent metric algebras that are needed for the proof. Some of the results in Section 6.4 about semisimple derivations of nilpotent algebras may be of independent interest.

In Section 7 the solution spaces to linear systems of the form $U v=$ $-2 \beta[1]_{m \times 1}$ are analyzed. It is shown in Theorem 12 that if a metric algebra $\left(\mathfrak{n}_{\mu}, Q\right)$ with Ricci eigenvector basis $\mathcal{B}$ has the property that $\alpha_{i j}^{j}=0$ for all $i$ and $j$, then the Gram matrix $U$ can be written in the form $U=c A+d[1]_{m \times m}$ where $A$ is a generalized Cartan matrix and $c$ and $d$ are positive. We call $A$ an associated matrix for $U$. Theorem 2 characterizes the solution spaces for the linear systems $U v=\left(c A+d[1]_{m \times m}\right) v=-2 \beta[1]_{m \times 1}$ by the properties of a generalized Cartan matrix $A$ associated to $U$. We recall that an irreducible generalized Cartan matrix is one of three types: finite type, affine type, or indefinite type. For a column vector $u=\left(u_{1}, u_{2}, \ldots,\right)^{T}$ we write $u>0$ $(u \geq 0)$ to denote that $u_{i}>0\left(u_{i} \geq 0\right)$ for all $i$.

Theorem 2. Let $A$ be a $m \times m$ irreducible generalized Cartan matrix and let $U=c A+d[1]_{m \times m}$, where $c$ and $d$ are positive. Suppose $\beta<0$. Then there exists a unique real number $\mu$ such that solutions to

$$
U v=-2 \beta[1]_{m \times 1}
$$

are the same as solutions to

$$
A v=\mu[1]_{m \times 1} .
$$

There are five mutually exclusive possibilities for the common solution space: 
(Fin) $A$ is of finite type, $\mu>0$, Equation (3) has a unique solution $v$, and $v>0$.

(Aff) $A$ is of affine type, $\mu=0$, Equation (3) has a unique solution $v$, and $v>0$.

(Ind1) $A$ is of indefinite type, $\mu<0$, Equation (3) has a positive solution $v_{0}$, the general solution to Equation (3) is

$$
v_{0}+\operatorname{ker} A=v_{0}+\operatorname{ker} U=v_{0}+\operatorname{ker} Y^{T},
$$

and the intersection of the solution space and the cone $\{v \in$ $\left.\mathbb{R}^{m} \mid v \geq 0\right\}$ is a simplex of dimension $\operatorname{ker} A$ parallel to $[1]_{m \times 1}^{\perp}$.

(Ind2) $A$ is of indefinite type, $\mu<0$ and Equation (3) is consistent but has no solution $v$ with $v>0$.

(Ind3) $A$ is of indefinite type, $\mu<0$, and Equation (3) is not consistent.

Additionally, if $U$ is the Gram matrix for a metric algebra $\left(\mathfrak{n}_{\mu}, Q\right)$ with respect to a Ricci eigenvector basis $\mathcal{B}$, then Case (Ind3) can not occur.

Each of the first four cases actually occurs in systems arising from metric nilpotent Lie algebras; we present examples of each type.

In Section 8 we consider parameter spaces of metric nilpotent Lie algebras. Before we state the main result of the section, we define some terminology. Fix an orthonormal basis $\mathcal{B}$ of an $n$-dimensional inner product space $(\mathfrak{n}, Q)$. Let $\mathcal{R}(\mathfrak{n}, \mathcal{B})$ be the subset of $\Lambda^{2} \mathfrak{n}^{*} \otimes \mathfrak{n}$ consisting of all $\mu$ so that $\mathcal{B}$ is a Ricci eigenvector basis for $\left(\mathfrak{n}_{\mu}, Q\right)$. Every $n$ dimensional metric algebra is metrically isomorphic to $\left(\mathfrak{n}_{\mu}, Q\right)$ for some $\mu$ in $\mathcal{R}(\mathfrak{n}, \mathcal{B})$ because Ricci eigenvector bases always exist.

We let $\mathcal{T}(\mathfrak{n}, \mathcal{B})$ be the set of all nontrivial elements $\mu$ of that $\Lambda^{2} \mathfrak{n}^{*} \otimes \mathfrak{n}$ have the property that $\Lambda\left(\mathfrak{n}_{\mu}, \mathcal{B}\right)$ is a subset of $\Theta_{n}$. We will say that an element of $\mathcal{T}(\mathfrak{n}, \mathcal{B})$ is $\mathcal{B}$-triangular. Note that for all $\mu$ in $\mathcal{T}(\mathfrak{n}, \mathcal{B})$, the algebra $\mathfrak{n}_{\mu}$ is nilpotent. Every $n$-dimensional metric algebra satisfying the nilsoliton condition is represented by an element of $\mathcal{R}(\mathfrak{n}, \mathcal{B}) \cap \mathcal{T}(\mathfrak{n}, \mathcal{B})$ (by Corollary 18 in Section 2.1).

Let $\mathcal{L}(\mathfrak{n}, \mathcal{B})$ be the subset of $\Lambda^{2} \mathfrak{n}^{*} \otimes \mathfrak{n}$ of Lie algebras: the algebraic variety consisting of those $\mu$ so that $\mathfrak{n}_{\mu}$ satisfies the Jacobi identity. The general linear group $G L_{n}(\mathbb{R})$ acts on $\Lambda^{2} \mathfrak{n}^{*} \otimes \mathfrak{n}$ by $(g \cdot \mu)(X, Y)=$ $g \mu\left(g^{-1} X, g^{-1} Y\right)$. Two Lie brackets $\mu$ in $\mathcal{L}(\mathfrak{n}, \mathcal{B})$ define isomorphic Lie algebras if they are in the same $G L_{n}(\mathbb{R})$ orbit. Hence the space of $n$-dimensional Lie algebras can be identified with quotient space of $\mathcal{L}(\mathfrak{n}, \mathcal{B})$ under this $G L_{n}(\mathbb{R})$ action.

Fix an orthonormal basis for the metric algebra $\left(\mathfrak{n}_{\mu}, Q\right)$. The set $\Lambda^{2} \mathfrak{n}^{*} \otimes \mathfrak{n}$ is partitioned into equivalence classes $\Omega_{\Lambda}$ that are level sets for the function assigning an element $\mu$ to the set $\Lambda\left(\mathfrak{n}_{\mu}, \mathcal{B}\right)$ of nonzero 
structure constants for $\left(\mathfrak{n}_{\mu}, Q\right)$ with respect to the basis $\mathcal{B}$. When $\Lambda=\emptyset$, we call $\Omega_{\Lambda}$ trivial. For each element $\mu$ in a nontrivial equivalence class $\Omega_{\Lambda}$, the root matrix $Y$ and Gram matrix $U$ for $\left(\mathfrak{n}_{\mu}, Q\right)$ with respect to $\mathcal{B}$ are the same, as they depend only on the common set $\Lambda\left(\mathfrak{n}_{\mu}, \mathcal{B}\right)$. This partition restricts to a partition of $\mathcal{T}(\mathfrak{n}, \mathcal{B})$ because we can write $\mathcal{T}(\mathfrak{n}, \mathcal{B})$ as the union $\cup_{\varnothing \neq \Lambda \subset \Theta_{n}} \Omega_{\Lambda}$ of equivalence classes $\Omega_{\Lambda}$ for all nontrivial subsets $\Lambda$ of $\Theta_{n}$.

The description of the set of metric algebras with a specified set of nonzero structure constants that satisfy the nilsoliton condition is given in the next theorem.

Theorem 3. Let $(\mathfrak{n}, Q)$ be an inner product space with orthonormal basis $\mathcal{B}$. Let $\Omega_{\Lambda} \subset \Psi_{n}$. Let $U$ denote the Gram matrix for elements of $\Omega_{\Lambda}$ and let $m=\|\Lambda\|$. Suppose $\beta<0$. Then the set of metric algebras $\left(\mathfrak{n}_{\mu}, Q\right)$ in $\Omega_{\Lambda}$ whose structure vector satisfies $U\left[\alpha^{2}\right]=-2 \beta[1]$ is

- finite of size $2^{m}$, if $U$ is nonsingular and there is a unique positive solution to $U v=-2 \beta[1]$,

- a finite disjoint union of the interiors of $2^{m}$ simplices of dimension $\operatorname{ker} U$ if $U$ is singular and $U v=-2 \beta[1]$ has a solution $v>0$, or

- empty if there are no solutions to $U v=-2 \beta[1]$ with $v>0$.

Also, the endomorphism $\mathrm{Ric}_{\mu}$ is the same for all elements $\mu$ of $\Omega_{\Lambda} \cap$ $\mathcal{R}(\mathfrak{n}, \mathcal{B})$ such that $U\left[\alpha^{2}\right]_{\mu}=-2 \beta[1]$.

In Section 9, we present examples of nilsoliton metrics constructed by solving equations of the form $U v=[1]$. The linear systems associated to the higher rank symmetric space $\mathcal{P}_{n}=S L_{n}(\mathbb{R}) / S O(n)$, the quaternionic hyperbolic space $H^{2}(\mathbb{H})$ and the Cayley plane are computed and solved, yielding continuous families of Einstein solvmanifolds around the latter two symmetric spaces and around $\mathcal{P}_{n}$ for $n \geq 5$. Linear systems $U v=[1]$ for the family of the simplest filiform Lie algebras, those with a codimension one abelian ideal, are computed and used to prove in Theorem 25 the existence of nilsoliton metrics unique up to scaling on these Lie algebras in each dimension, adding to the analytic proof of this theorem by J. Lauret from Lau02] a new algebraic proof. We address the question of which metric nilpotent Lie algebras admit symmetric derivations in Section 6.4, and state a necessary combinatorial condition. In Theorem 32 we give an example of a metric nilpotent Lie algebra that admits a positive definite derivation but does not admit a nilsoliton metric; this shows that characteristic nilpotence is not the only obstruction to the existence of a a nilsoliton metric on a nilpotent Lie algebra. 
In Section 10, we address the question of when the sum of two nilsoliton structures on an inner product space is nilsoliton. For a symmetric map $D$ of an $n$-dimensional vector space with a basis of $D$-eigenvectors $\mathcal{B}=\left\{X_{i}\right\}_{i=1}^{n}$, we define the eigenvalue vector $v_{D}$ of $D$ with respect to $\mathcal{B}$ to be $v_{D}=\left(\mu_{1}, \ldots, \mu_{n}\right)^{T}$, where $\mu_{i}$ is the eigenvalue of $X_{i}$ for $i=1, \ldots, n$. For a $n$-dimensional metric algebra $\left(\mathfrak{n}_{\mu}, Q\right)$ with orthonormal basis $\mathcal{B}$, define the Ricci vector to be

$$
\operatorname{Ric}_{\mu}^{\mathcal{B}}=\left(\operatorname{ric}_{\mu}\left(X_{1}, X_{1}\right), \operatorname{ric}_{\mu}\left(X_{2}, X_{2}\right), \ldots, \operatorname{ric}_{\mu}\left(X_{n}, X_{n}\right)\right)^{T} .
$$

When $\mathcal{B}$ is an Ricci eigenvector basis, the Ricci vector is the eigenvalue vector $v_{\text {Ric }}$ for the Ricci endomorphism (recall that Ricci eigenvector bases are always assumed to be orthonormal). We establish a sufficient condition, formulated in terms of Ricci vectors, for the sum of metric algebraic structures $\mu_{1}$ and $\mu_{2}$ on an inner product space $(\mathfrak{n}, Q)$ to satisfy the nilsoliton condition:

Theorem 4. Let $(\mathfrak{n}, Q)$ be an inner product space with orthonormal basis $\mathcal{B}$. Let $\mu_{1}$ and $\mu_{2}$ be nontrivial elements of $\mathcal{R}(\mathfrak{n}, \mathcal{B})$ defining metric algebras $\left(\mathfrak{n}_{\mu_{1}}, Q\right)$ and $\left(\mathfrak{n}_{\mu_{2}}, Q\right)$ satisfying the nilsoliton condition with nilsoliton constants $\beta_{1}$ and $\beta_{2}$ respectively. Suppose that the set $\Lambda\left(\mathfrak{n}_{\mu_{1}+\mu_{2}}, \mathcal{B}\right)$ is the disjoint union of the sets $\Lambda\left(\mathfrak{n}_{\mu_{1}}, \mathcal{B}\right)$ and $\Lambda\left(\mathfrak{n}_{\mu_{2}}, \mathcal{B}\right)$. If there are constants $c_{1}$ and $c_{2}$ so that $Y_{i j}^{k} \mathbf{R i c}_{\mu_{1}}^{\mathcal{B}}=c_{1}$ for all $(i, j, k)$ in $\Lambda\left(\mathfrak{n}_{\mu_{2}}, \mathcal{B}\right), Y_{i j}^{k} \mathbf{R i c}_{\mu_{2}}^{\mathcal{B}}=c_{2}$ for all $(i, j, k)$ in $\Lambda\left(\mathfrak{n}_{\mu_{1}}, \mathcal{B}\right)$, and $-2 \beta_{1}+c_{2}=-2 \beta_{2}+c_{1}=: \beta$, then $\left(\mathfrak{n}_{\mu_{1}+\mu_{2}}, Q\right)$ satisfies the nilsoliton condition with nilsoliton constant $\beta$.

We use this theorem in the proof of Theorem 35 which asserts the existence of nilsoliton metrics on each member of an infinite family $Q_{n}$ of filiform metric nilpotent Lie algebras. Combining the existence results in Theorems 25 and 35, we conclude in Corollary 36 that every naturally graded filiform nilpotent Lie algebra admits a nilsoliton metric.

\section{Preliminaries}

2.1. The geometry of metric nilpotent Lie algebras. Let $(N, g)$ be a nilmanifold with associated metric Lie algebra $(\mathfrak{n}, Q)$. We will denote by $X$ both a vector in $\mathfrak{n}$ and the left-invariant vector field on $N$ that it induces. The connection for $(N, g)$ is given by

$$
\nabla_{X} Y=\frac{1}{2}\left(\operatorname{ad}_{X} Y-\operatorname{ad}_{X}^{*} Y-\operatorname{ad}_{Y}^{*} X\right) \text {, }
$$

and the sectional curvature $K$ of a plane spanned by orthonormal $X$ and $Y$ is

$$
K(X \wedge Y)=\left\|\nabla_{X} Y\right\|^{2}-\left\langle\nabla_{X} X, \nabla_{Y} Y\right\rangle-\left\langle\operatorname{ad}_{Y}^{2} X, X\right\rangle-\left\|\operatorname{ad}_{X} Y\right\|^{2}
$$


([Wol91], Ale75]). Let $\left\{X_{i}\right\}$ be an orthonormal basis of $\mathfrak{n}$. We view the Ricci form for $(N, g)$ as in inner product on $\mathfrak{n}$ and the Ricci endomorphism as an linear map from $\mathfrak{n}$ to itself. The Ricci form ric for $(N, g)$ is given by the expression for the nil-Ricci form in Equation (11), where we denote the metric $g$ by $\langle\cdot, \cdot\rangle$. This is a special case of the the general formula for the Ricci form for a homogeneous space (Corollary 7.38, [Bes87]).

\subsection{Generalized Cartan matrices and their Dynkin diagrams.}

The following background material is from Chapter 4 of [Kac90], where it is used to study root systems of Kac-Moody algebras, generalizing classical results for semisimple Lie algebras.

A real $n \times n$ matrix $A=\left(a_{i j}\right)_{i, j=1}^{n}$ is called a generalized Cartan matrix if

(1) $a_{i i}=2$ for $i=1, \ldots, n$,

(2) $a_{i j}$ are nonpositive integers for $i \neq j$, and

(3) if $a_{i j}=0$, then $a_{j i}=0$

A matrix is decomposable if it is block diagonal after conjugation by a permutation matrix, and is indecomposable if it is not decomposable. A vector $v$ will be called positive if $v>0$.

Theorem 5 ([Kac90]). Let $A$ be a real $n \times n$ indecomposable matrix so that $a_{i j}$ is nonpositive if $i \neq j$ and $a_{i j}=0$ implies that $a_{j i}=0$. Then one and only one of the following three possibilities holds for both $A$ and $A^{T}$ :

(Fin) $\operatorname{det} A \neq 0$; there exists $u>0$ such that $A u>0$; and if $A v \geq 0$ then $v>0$ or $v=0$.

(Aff) $\operatorname{dim} \operatorname{ker} A=1$; there exists $u>0$ such that $A u=0$; and if $A v \geq 0$ then $A v=0$.

(Ind) there exists $u>0$ such that $A u<0$; and if $A v \geq 0$ and $v \geq 0$, then $v=0$.

These three cases are called finite type, affine type and indefinite type respectively. The Dynkin diagrams of all generalized Cartan matrices of finite and affine type are classified.

\section{Curvatures for metric nilpotent Algebras}

3.1. Ricci curvature. We often want to represent the nil-Ricci endomorphism for a given metric algebra $(\mathfrak{n}, Q)$ with respect to a basis. Corollary 7 allows us to check whether a basis is a Ricci eigenvector basis. Once we have a Ricci eigenvector basis, Theorem 8 will give the eigenvalues for each of the basis vectors, thus determining the endomorphism. 
For a metric algebra $\left(\mathfrak{n}_{\mu}, Q\right)$, the linear map $J: \mathfrak{n} \rightarrow \operatorname{End}(\mathfrak{n})$ is defined by $J_{X} Y=\operatorname{ad}_{Y}^{*} X$; it encodes the algebraic structure of $\mathfrak{n}_{\mu}$ using the inner product $Q$. The inner product $\langle\cdot, \cdot\rangle$ on $\mathfrak{n}$ induces an inner product on the tensor algebra of $\mathfrak{n}$, which we also denote by $\langle\cdot, \cdot\rangle$. In the next theorem, we use this inner product to write the Ricci form for $\left(\mathfrak{n}_{\mu}, Q\right)$ in terms of the adjoint and $J$ maps in $\operatorname{End}(\mathfrak{n})$.

Theorem 6. Suppose that $\left(\mathfrak{n}_{\mu}, Q\right)$ is a nilpotent metric algebra. Then

$$
\operatorname{ric}_{\mu}(X, Y)=-\frac{1}{2}\left\langle\operatorname{ad}_{X}, \operatorname{ad}_{Y}\right\rangle+\frac{1}{4}\left\langle J_{X}, J_{Y}\right\rangle
$$

for any $X, Y \in \mathfrak{n}$.

Proof. Let $\left\{X_{i}\right\}_{i=1}^{n}$ be an orthonormal basis for $\left(\mathfrak{n}_{\mu}, Q\right)$. From Equation (11) we get

$$
\begin{aligned}
& \operatorname{ric}_{\mu}(X, Y) \\
& =-\frac{1}{2} \sum_{i=1}^{n}\left\langle\operatorname{ad}_{X} X_{i}, \operatorname{ad}_{Y} X_{i}\right\rangle+\frac{1}{4} \sum_{i, j=1}^{n}\left\langle\operatorname{ad}_{X_{i}} X_{j}, X\right\rangle\left\langle\operatorname{ad}_{X_{i}} X_{j}, Y\right\rangle \\
& =-\frac{1}{2} \sum_{i, j=1}^{n}\left\langle\operatorname{ad}_{X} X_{i}, X_{j}\right\rangle\left\langle\operatorname{ad}_{Y} X_{i}, X_{j}\right\rangle \\
& \quad+\frac{1}{4} \sum_{i, j=1}^{n}\left\langle X_{j}, \operatorname{ad}_{X_{i}}^{*} X\right\rangle\left\langle X_{j}, \operatorname{ad}_{X_{i}}^{*} Y\right\rangle \\
& =-\frac{1}{2}\left\langle\operatorname{ad}_{X}, \operatorname{ad}_{Y}\right\rangle+\frac{1}{4} \sum_{i, j=1}^{n}\left\langle X_{j}, J_{X} X_{i}\right\rangle\left\langle X_{j}, J_{Y} X_{i}\right\rangle \\
& =-\frac{1}{2}\left\langle\operatorname{ad}_{X}, \operatorname{ad}_{Y}\right\rangle+\frac{1}{4}\left\langle J_{X}, J_{Y}\right\rangle
\end{aligned}
$$

q.e.d.

This has a corollary that will be useful in concrete situations.

Corollary 7. Let $\left(\mathfrak{n}_{\mu}, Q\right)$ be an n-dimensional metric algebra. Then an orthonormal basis $\left\{X_{i}\right\}_{i=1}^{n}$ is a Ricci eigenvector basis if $\left\{J_{X_{i}}\right\}_{i=1}^{n}$ and $\left\{\operatorname{ad}_{X_{i}}\right\}_{i=1}^{n}$ are both orthogonal sets in $\operatorname{End}(\mathfrak{n})$.

If $\left\{J_{X_{i}}\right\}_{i=1}^{n}\left\{\operatorname{ad}_{X_{i}}\right\}_{i=1}^{n}$ are both orthogonal sets in $\operatorname{End}(\mathfrak{n})$, then $\mathcal{B}$ is a Ricci eigenvector basis by the corollary above. The orthogonality of these sets can sometimes be verified simply by looking at how elements on $\Lambda(\mathfrak{n}, \mathcal{B})$ overlap. For example, triples of form $(1,2,3)$ and $(1,2,4)$ in $\Lambda(\mathfrak{n}, \mathcal{B})$ mean that $J_{X_{3}}$ and $J_{X_{4}}$ may not be orthogonal, and triples $(1,3,4)$ and $(2,3,4)$ mean that $\operatorname{ad}_{X_{1}}$ and $\operatorname{ad}_{X_{2}}$ may not be orthogonal. 
The lack of such "overlapping triples" will imply that the sets are orthogonal.

Given an inner product space $\left(\mathfrak{n}_{\mu}, Q\right)$ with orthonormal basis $\mathcal{B}$ we can use the corollary to describe membership in $\mathcal{R}(\mathfrak{n}, \mathcal{B})$ with polynomial equations in the structure constants; therefore, $\mathcal{R}(\mathfrak{n}, \mathcal{B})$ is an algebraic variety in $\Lambda^{2} \mathfrak{n}^{*} \otimes \mathfrak{n}$.

Let $\mathcal{B}=\left\{X_{i}\right\}_{i=1}^{n}$ be a Ricci eigenvector basis for a metric algebra $\left(\mathfrak{n}_{\mu}, Q\right)$. Next we show that the transpose of the Ricci vector is a weighted sum of the root vectors for $\left(\mathfrak{n}_{\mu}, Q\right)$ with respect to $\mathcal{B}$, where the weight of a root vector is $-1 / 2$ times the square of the corresponding structure constant. This will be illustrated in Example 10. The formula was first given as Proposition 4.1 of [Kar93, where R. Karidi gave a detailed analysis of the effects of individual structure constants on the curvature tensor of a metric nilpotent Lie algebra.

Theorem 8. Let $\left(\mathfrak{n}_{\mu}, Q\right)$ be a metric algebra and let $\mathcal{B}=\left\{X_{i}\right\}_{i=1}^{n}$ be a Ricci eigenvector basis for $\mathfrak{n}_{\mu}$. Let $\left\{Y_{i j}^{k} \mid(i, j, k) \in \Lambda(\mathfrak{n}, \mathcal{B})\right\}$ be the set of root vectors for $\left(\mathfrak{n}_{\mu}, Q\right)$ with respect to $\mathcal{B}$, and let $Y$ be the root matrix. Then the eigenvalues $\kappa_{X_{1}}, \kappa_{X_{2}}, \ldots, \kappa_{X_{n}}$ of the nil-Ricci endomorphism are given by

$$
\begin{aligned}
\boldsymbol{R i c}_{\mu}^{\mathcal{B}} & =\left(\kappa_{X_{1}}, \kappa_{X_{2}}, \ldots, \kappa_{X_{n}}\right)^{T} \\
& =-\frac{1}{2} \sum_{(i, j, k) \in \Lambda}\left(\alpha_{i j}^{k}\right)^{2}\left(Y_{i j}^{k}\right)^{T}=-\frac{1}{2} Y^{T}\left[\alpha^{2}\right] .
\end{aligned}
$$

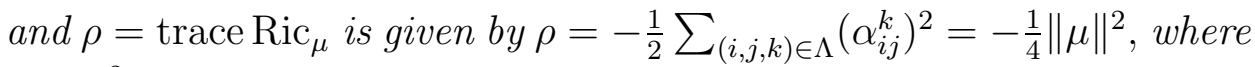
$\mu \in \Lambda^{2} \mathfrak{n}^{*} \otimes \mathfrak{n}$ is the Lie bracket for $\mathfrak{n}$.

When $\left(\mathfrak{n}_{\mu}, Q\right)$ is a metric nilpotent Lie algebra, the number $\rho$ is the scalar curvature for corresponding nilmanifold.

Proof. We use Equation (1) to express the eigenvalue vector for the Ricci endomorphism.

$$
\begin{aligned}
\sum_{k=1}^{n} \operatorname{ric}_{\mu}\left(X_{k}, X_{k}\right) \varepsilon_{k}^{T} & \\
= & -\frac{1}{2} \sum_{i, k=1}^{n}\left\|\left[X_{k}, X_{i}\right]\right\|^{2} \varepsilon_{k}^{T}+\frac{1}{4} \sum_{i, j, k=1}^{n}\left\langle\left[X_{i}, X_{j}\right], X_{k}\right\rangle^{2} \varepsilon_{k}^{T} \\
& =-\frac{1}{2} \sum_{i, j, k=1}^{n}\left\langle\left[X_{k}, X_{i}\right], X_{j}\right\rangle^{2} \varepsilon_{k}^{T}+\frac{1}{4} \sum_{i, j, k=1}^{n}\left\langle\left[X_{i}, X_{j}\right], X_{k}\right\rangle^{2} \varepsilon_{k}^{T}
\end{aligned}
$$


Breaking the first summand into two pieces and reindexing the first two pieces, we see this equals

$$
\begin{aligned}
=- & \frac{1}{4} \sum_{i, j, k=1}^{n}\left\langle\left[X_{i}, X_{j}\right], X_{k}\right\rangle^{2} \varepsilon_{i}^{T}-\frac{1}{4} \sum_{i, j, k=1}^{n}\left\langle\left[X_{i}, X_{j}\right], X_{k}\right\rangle^{2} \varepsilon_{j}^{T} \\
& +\frac{1}{4} \sum_{i, j, k=1}^{n}\left\langle\left[X_{i}, X_{j}\right], X_{k}\right\rangle^{2} \varepsilon_{k}^{T} \\
= & -\frac{1}{4} \sum_{i, j, k=1}^{n}\left(\alpha_{i j}^{k}\right)^{2} Y_{i j}^{k} \\
= & -\frac{1}{2} \sum_{1 \leq i<j \leq n} \sum_{k=1}^{n}\left(\alpha_{i j}^{k}\right)^{2} Y_{i j}^{k}
\end{aligned}
$$

which when summed over nonzero terms becomes

$$
=-\frac{1}{2} \sum_{(i, j, k) \in \Lambda}\left(\alpha_{i j}^{k}\right)^{2} Y_{i j}^{k}
$$

Taking the transpose of both sides we get

$$
\operatorname{Ric}_{\mu}^{\mathcal{B}}=-\frac{1}{2} \sum_{(i, j, k) \in \Lambda}\left(\alpha_{i j}^{k}\right)^{2}\left(Y_{i j}^{k}\right)^{T},
$$

completing the proof.

q.e.d.

3.2. Sectional curvature. A Lie algebra $\mathfrak{g}$ is said to be $\mathbb{R}^{+}$-graded if it can be written as $\mathfrak{g}=\mathfrak{g}_{\lambda_{1}} \oplus \cdots \oplus \mathfrak{g}_{\lambda_{r}}$ where $\left[\mathfrak{g}_{\lambda_{i}}, \mathfrak{g}_{\lambda_{j}}\right] \subset \mathfrak{g}_{\lambda_{i}+\lambda_{j}}$ $\left(\mathfrak{g}_{\lambda_{k}}:=0\right.$ if $\left.\lambda_{k}>\lambda_{r}\right)$ and $\lambda_{1}, \lambda_{2}, \ldots, \lambda_{r}$ are in $\mathbb{R}^{+}$. An $\mathbb{R}^{+}$-graded algebra is nilpotent. For a nilmanifold, tangent two-planes spanned by two vectors in subspace summands of a $\mathbb{R}^{+}$-grading, the sectional curvature has a particularly nice expression.

Theorem 9. Let $(\mathfrak{n}, Q)$ be a metric Lie algebra. Suppose that $\mathfrak{n}$ has a orthogonal $\mathbb{R}^{+}$-grading $\mathfrak{n}=\oplus \mathfrak{n}_{\lambda}$. For unit $X$ in $\mathfrak{n}_{\mu}$ and unit $Y$ in $\mathfrak{n}_{\nu}$ orthogonal to $X$, if $\mu \leq \nu$, the sectional curvature of the plane spanned by $X$ and $Y$ is given by

$$
K(X \wedge Y)=-\frac{3}{4}\left\|\operatorname{ad}_{X} Y\right\|^{2}+\frac{1}{4}\left\|J_{Y} X\right\|^{2} .
$$

If in addition $X=X_{i}, Y=Y_{j}$ for an orthonormal basis $\left\{X_{i}\right\}_{i=1}^{n}$, then

$$
K\left(X_{i} \wedge X_{j}\right)=-\frac{3}{4} \sum_{(i, j, k) \in \Lambda(\mathfrak{n}, \mathcal{B})}\left(\alpha_{i j}^{k}\right)^{2}+\frac{1}{4} \sum_{(i, k, j) \in \Lambda(\mathfrak{n}, \mathcal{B})}\left(\alpha_{i j}^{k}\right)^{2} .
$$


The positive definite derivation $\mathrm{Ric}-\beta$ Id for a metric nilpotent algebra satisfying the nilsoliton condition with nilsoliton constant $\beta<0$ defines an $\mathbb{R}^{+}$grading, so we can use the previous theorem to find sectional curvatures for nilsolitons.

Proof. Suppose that $X$ and $Y$ are orthogonal unit vectors in $\mathfrak{g}_{\mu}$ and $\mathfrak{g}_{\nu}$ respectively, and $\mu \leq \nu$. Since $\oplus \mathfrak{g}_{\lambda}$ is a grading, $\operatorname{ad}_{X} Y$ is in $\mathfrak{g}_{\mu+\nu}$. We claim that for any $W$ in any $\mathfrak{g}_{\lambda}$, the vector $J_{Y} X$ is in $\mathfrak{g}_{\nu-\mu}$. The inner product $\left\langle Y, \operatorname{ad}_{X} W\right\rangle=\left\langle J_{Y} X, W\right\rangle$ is nonzero only if $\nu=\mu+\lambda$, by the orthogonality of the grading. This shows that $J_{Y} X$ is in $\mathfrak{g}_{\nu-\mu}$. The same argument shows that $J_{X} Y=0$ since $\nu-\mu \leq 0$ makes $\mathfrak{g}_{\mu-\nu}$ trivial.

Now we use Equation (6) to compute

$$
\begin{aligned}
\left\|\nabla_{X} Y\right\|^{2} & =\frac{1}{4}\left\langle\operatorname{ad}_{X} Y-J_{Y} X, \operatorname{ad}_{X} Y-J_{Y} X\right\rangle \\
& =\frac{1}{4}\left(\left\|\operatorname{ad}_{X} Y\right\|^{2}-2\left\langle\operatorname{ad}_{X} Y, J_{Y} X\right\rangle+\left\|J_{Y} X\right\|^{2}\right) \\
& =\frac{1}{4}\left(\left\|\operatorname{ad}_{X} Y\right\|^{2}+\left\|J_{Y} X\right\|^{2}\right)
\end{aligned}
$$

The term $\left\langle\operatorname{ad}_{X} Y, J_{Y} X\right\rangle$ vanishes since $\operatorname{ad}_{X} Y$ is in $\mathfrak{g}_{\mu+\nu}$ and $J_{Y}$ is in $\mathfrak{g}_{\nu-\mu}$, and these orthogonal spaces can not coincide unless $\mu=0$. Equation (6) also shows that $\nabla_{W} W=0$ for any $W$ in any $\mathfrak{g}_{\lambda}$ because $\operatorname{ad}_{X}^{*} X=J_{X} X$ is in $\mathfrak{g}_{0}=\{0\}$.

Substituting the expressions for $\nabla_{X} X, \nabla_{Y} Y$ and $\left\|\nabla_{X} Y\right\|^{2}$ into Equation (7) and simplifying, we get

$$
\begin{aligned}
K(X \wedge Y) & =\frac{1}{4}\left(\left\|\operatorname{ad}_{X} Y\right\|^{2}+\left\|J_{Y} X\right\|^{2}\right)-\left\|\operatorname{ad}_{X} Y\right\|^{2} \\
& =-\frac{3}{4}\left\|\operatorname{ad}_{X} Y\right\|^{2}+\frac{1}{4}\left\|J_{Y} X\right\|^{2}
\end{aligned}
$$

as desired. The equation for $K\left(X_{i} \wedge X_{j}\right)$ follows from writing the equation for $K(X \wedge Y)$ relative to an orthonormal basis and setting terms not in $\Lambda\left(\mathfrak{n}_{\mu}, Q\right)$ equal to zero.

q.e.d.

3.3. Examples. We illustrate the preceding theorems with an example.

Example 10. Let $\left(\mathfrak{n}_{\mu}, Q\right)$ be the metric nilpotent Lie algebra with orthonormal basis $\mathcal{B}=\left\{X_{1}, X_{2}, X_{3}, X_{4}, X_{5}\right\}$ and bracket relations

$$
\left[X_{1}, X_{2}\right]=a X_{3},\left[X_{1}, X_{3}\right]=b X_{4},\left[X_{1}, X_{4}\right]=c X_{5},\left[X_{2}, X_{3}\right]=d X_{5},
$$

where $\mu$ is a function of $a, b, c, d \in \mathbb{R} \backslash\{0\}$. Here we have

$$
\Lambda(\mathfrak{n}, \mathcal{B})=\{(1,2,3),(1,3,4),(1,4,5),(2,3,5)\} .
$$

Notice that from the way triples in $\Lambda(\mathfrak{n}, \mathcal{B})$ overlap, the sets $\left\{\operatorname{ad}_{X_{i}}\right\}_{i=1}^{5}$ and $\left\{J_{X_{i}}\right\}_{i=1}^{5}$ are both orthogonal in $\operatorname{End}(\mathfrak{n})$ for all $a, b, c, d$, and then by Corollary $7,\left\{X_{i}\right\}_{i=1}^{5}$ is a Ricci eigenvector basis. The root matrix 
for $\left(\mathfrak{n}_{\mu}, Q\right)$ with respect to $\mathcal{B}$ is $Y=\left[\begin{array}{ccccc}1 & 1 & -1 & 0 & 0 \\ 1 & 0 & 1 & -1 & 0 \\ 1 & 0 & 0 & 1 & -1 \\ 0 & 1 & 1 & 0 & -1\end{array}\right]$ and the structure vector is $\left[\alpha^{2}\right]=\left(a^{2}, b^{2}, c^{2}, d^{2}\right)^{T}$. By Theorem 8 , the Ricci vector $\mathbf{R i c}_{\mu}^{\mathcal{B}}$ is given by

$$
\begin{aligned}
& -\frac{1}{2}\left(a^{2} Y_{12}^{3}+b^{2} Y_{13}^{4}+c^{2} Y_{14}^{5}+d^{2} Y_{23}^{5}\right)^{T}= \\
& \quad-\frac{1}{2}\left(a^{2}+b^{2}+c^{2}, a^{2}+d^{2},-a^{2}+b^{2}+d^{2},-b^{2}+c^{2},-c^{2}-d^{2}\right)^{T},
\end{aligned}
$$

and hence the eigenvalues for the Ricci endomorphism are

$$
-\frac{1}{2}\left(a^{2}+b^{2}+c^{2}\right), \frac{1}{2}\left(a^{2}+d^{2}\right), \frac{1}{2}\left(a^{2}-b^{2}-d^{2}\right), \frac{1}{2}\left(b^{2}-c^{2}\right), \frac{1}{2}\left(c^{2}+d^{2}\right)
$$

Thus, with respect to the basis $\mathcal{B}$, the Ricci endomorphism is represented by the diagonal matrix $\operatorname{diag}\left(\mathbf{R i c}_{\mu}^{\mathcal{B}}\right)$.

Notice that $\mathfrak{n}$ has a grading defined by $\mathfrak{n}=\oplus_{i=1}^{5} \mathfrak{n}_{i}$ with $\mathfrak{n}_{i}=\left\langle X_{i}\right\rangle$ for $i=1, \ldots, 5$. As an illustration of Theorem 9, we note that the sectional curvature of the plane spanned by $X_{1}$ and $X_{4}$ is $\frac{1}{4}\left(b^{2}-3 c^{2}\right)$.

The Gram matrix for $\Lambda(\mathfrak{n}, \mathcal{B})$ is

$$
U=Y Y^{T}=\left[\begin{array}{llll}
3 & 0 & 1 & 0 \\
0 & 3 & 0 & 1 \\
1 & 0 & 3 & 1 \\
0 & 1 & 1 & 3
\end{array}\right]
$$

and an associated generalized Cartan matrix is

$$
A=U-[1]_{4 \times 4}=\left[\begin{array}{cccc}
2 & -1 & 0 & -1 \\
-1 & 2 & -1 & 0 \\
0 & -1 & 2 & 0 \\
-1 & 0 & 0 & 2
\end{array}\right]
$$

It may be checked that the graphs $S(U)$ and $S(A)$ determined by $U$ and $A$ respectively both have the same incidences as type $A_{4}$. Note that because zero and nonzero off-diagonal entries are reversed in $U$ and $A$ that one graph can be easily obtained from the other.

In Lau02, using a variational approach, J. Lauret gave the first presentation of nonsymmetric Einstein solvmanifolds of arbitrarily high step, as extensions of nilsoliton Lie algebras of arbitrarily high step. In the next example, we use Theorem 8 to compute the Ricci curvature in this family. We will revisit this example later.

Example 11. Let $\mathfrak{n}_{\mu}$ be an $n$-dimensional metric nilpotent Lie algebra with a codimension one abelian ideal $\mathfrak{m}$. Let $X$ be a vector not in $\mathfrak{m}$. The mapping $\left.\operatorname{ad}_{X}\right|_{\mathfrak{m}}$ is nilpotent, so there exists a Jordan decomposition of 
$\mathfrak{m}$ as the direct sum of $r$ subspaces $\mathfrak{m}_{1}, \ldots, \mathfrak{m}_{r}$ of dimensions $n_{1}+1, n_{2}+$ $1, \ldots, n_{r}+1$ respectively, such that for each $i=1, \ldots, r$, there is a basis $\left\{X_{i j} \mid 1 \leq j \leq n_{i}+1\right\}$ for $\mathfrak{m}_{i}$ so that $\operatorname{ad}_{X}$ is given by $\left[X, X_{i j}\right]=a_{i j} X_{i(j+1)}$ $\left(X_{i\left(n_{1}+2\right)}:=0\right)$.

In terms of the lexicographically ordered basis $\left\{X_{i j}\right\}$, we may write $\left.\operatorname{ad}_{X}\right|_{\mathfrak{m}}$ as

$$
\left.\operatorname{ad}_{X}\right|_{\mathfrak{m}}=\left[\begin{array}{ccc}
J_{n_{1}} & & \\
& \ddots & \\
& & J_{n_{r}}
\end{array}\right], \quad \text { where } \quad J_{n_{i}}=\left[\begin{array}{cccc}
0 & & & \\
a_{i 1} & \ddots & & \\
& \ddots & \ddots & \\
& & a_{i n_{i}} & 0
\end{array}\right] \text {. }
$$

We impose the inner product $Q$ on $\mathfrak{n}_{\mu}$ that has $\mathcal{B}=\{X\} \cup\left\{X_{i j} \mid 1 \leq\right.$ $\left.i \leq r, 1 \leq j \leq n_{i}+1\right\}$ as an orthonormal basis. The basis $\mathcal{B}$ is a Ricci eigenvector basis by Corollary 7, because $\left\{\operatorname{ad}_{X_{i j}}\right\}$ and $\left\{J_{X_{i j}}\right\}$ are both orthogonal sets.

Observe that $J_{X} \equiv 0$, so by Theorem 6,

$$
\operatorname{ric}_{\mu}(X, X)=-\frac{1}{2}\left\|\operatorname{ad}_{X}\right\|^{2}=-\frac{1}{2} \sum_{1 \leq i \leq r} \sum_{1 \leq j \leq n_{i}+1} a_{i j}^{2} .
$$

Next we use Theorem 8 to find the restriction of the Ricci endomorphism to $\mathfrak{m}_{i}$ for each $i$. Using that $X_{i\left(n_{i}+2\right)}:=0$, we get that the projection of the Ricci vector $\mathbf{R i c}_{\mu}^{\mathcal{B}}$ to $\mathfrak{m}_{i}$ is

$$
\frac{1}{2}\left(-a_{i 1}^{2}, a_{i 1}^{2}-a_{i 2}^{2}, \ldots, a_{i\left(n_{i}-1\right)}^{2}-a_{i n_{i}}^{2}, a_{i n_{i}}^{2}\right)
$$

and hence

$$
\begin{aligned}
\operatorname{ric}_{\mu}\left(X_{i 1}, X_{i 1}\right) & =-\frac{1}{2} a_{i 1}^{2}, \\
\operatorname{ric}_{\mu}\left(X_{i j}, X_{i j}\right) & =\frac{1}{2}\left(a_{i(j-1)}^{2}-a_{i j}^{2}\right) \quad \text { for } j=2 \text { to } n_{i} \\
\operatorname{ric}_{\mu}\left(X_{i n_{i+1}}, X_{i n_{i+1}}\right) & =\frac{1}{2} a_{i n_{i}}^{2} .
\end{aligned}
$$

The Gram matrix $U$ and generalized Dynkin diagram $S(U)$ for $\left(\mathfrak{n}_{\mu}, Q\right)$ relative to $\mathcal{B}$ are not particularly nice; however the associated matrix $A=U-[1]_{n \times n}$ is of type $A_{n_{1}-1}+\cdots+A_{n_{r}-1}$.

\section{Properties of the Gram matrix $U$ and associated MATRIX $A$}

In the next proposition, we summarize important properties of the Gram matrix. 
Theorem 12. Let $(\mathfrak{n}, Q)$ be an $n$-dimensional inner product space with Ricci eigenvector basis $\mathcal{B}$. Let $\mu$ be a $\Lambda^{2} \mathfrak{n}^{*} \otimes \mathfrak{n}$ so that $\Lambda\left(\mathfrak{n}_{\mu}, \mathcal{B}\right) \subset \Psi_{n}$. Let $U=\left(u_{i j}\right)$ be the Gram matrix for $\left(\mathfrak{n}_{\mu}, Q\right)$ with respect to $\mathcal{B}$. Then

(1) The diagonal entries of $U$ are all three

(2) The off-diagonal entries of $U$ are in the set $\{-2,-1,0,1,2\}$.

(3) $U$ is symmetric

(4) The rank of $U$ is equal to the number of independent vectors in the set $\left\{Y_{i j}^{k} \mid(i, j, k) \in \Lambda(\mathfrak{n}, \mathcal{B})\right\}$ of root vectors for $\left(\mathfrak{n}_{\mu}, Q\right)$ with respect to $\mathcal{B}$.

(5) $U$ is positive semidefinite.

Furthermore, the associated matrix $A$ defined by $A=2 U-4[1]_{m \times m}$, where $m=\|\Lambda\|$, is a generalized Cartan matrix. If $U$ has no entries of 2 , then $A^{\prime}=U-[1]_{m \times m}$ is a generalized Cartan matrix. Also, $U$ is positive definite if either $A$ or $A^{\prime}$ is positive definite

Proof. By skew-symmetry and the hypothesis that $\Lambda \subset \Psi_{n}, \alpha_{i j}^{k}=0$ if $i, j$, and $k$ are not all distinct, so if $(i, j, k) \in \Lambda$, the structure vector $Y_{i j}^{k}$ is represented by a vector with two entries of one and one entry of -1 , with zeroes comprising the remaining entries. The $(i, j)$ entry of $U$ is the inner product of the $i$ th structure vector $Y_{i}$ and the $j$ th structure vector $Y_{j}$. After considering all the possibilities for the arrangements of the nonzero entries of $Y_{i}$ and $Y_{j}$, one sees that the inner product $\left\langle Y_{i}, Y_{j}\right\rangle$ must be in the set $\{-2,-1,0,1,2,3\}$, with value three if and only if $Y_{i}=Y_{j}$. As the root vectors are all distinct, this occurs if and only if $i=j$. Thus, properties (1) and (2) hold. The definition of $U$ as $Y Y^{T}$ insures that properties (3), (4) and (5) hold ([HJ90, Theorem 7.2.10).

Properties (1)-(3) of generalized Cartan matrices hold for $A$ from the definition of associated matrix, the symmetry of $U$, and the fact that $[1]_{m \times m}$ is a symmetric matrix. For any $n \times 1$ vector $v, v A v^{T}=$ $2 v U v^{T}-4 v v^{T}$ and $v A^{\prime} v^{T}=v U v^{T}-v v^{T}$ so if $v A v^{T}>0$ or $v A^{\prime} v^{T}>0$, then $v U v^{T}>0$.

q.e.d.

\section{The Jacobi CONDITION}

The next theorem gives a simple way to check if a set of nonzero structure constants indexed by a subset of the set $\Theta_{n}$ define a Lie algebra. We will use the theorem later to check whether a metric algebra $\mathfrak{n}_{\mu}$ satisfies the Jacobi condition when it is represented with respect to a triangular orthonormal basis. 
Theorem 13. Let $(\mathfrak{n}, Q)$ be an $n$-dimensional inner product space, and let $\mu$ be an element of $\Lambda^{2} \mathfrak{n}^{*} \otimes \mathfrak{n}$. Let $\mathcal{B}=\left\{X_{i}\right\}_{i=1}^{n}$ be an orthonormal basis for $(\mathfrak{n}, Q)$ and suppose that $\mu$ is $\mathcal{B}$-triangular. Then

(1) If $i, j$ and $k$ are distinct, the product $\alpha_{i j}^{l} \alpha_{l k}^{m} \neq 0$ if and only if $\left\langle Y_{i j}^{l}, Y_{l k}^{m}\right\rangle=-1$.

(2) The algebra $\mathfrak{n}_{\mu}$ defined by $\mu$ is a Lie algebra if and only if whenever there exists $m$ so that $\left\langle Y_{i j}^{l}, Y_{l k}^{m}\right\rangle=-1$ for triples $(i, j, l)$ and $(l, k, m)$ or $(k, l, m)$ in $\Lambda\left(\mathfrak{n}_{\mu}, \mathcal{B}\right)$, the equation

$$
\sum_{s<m} \alpha_{i j}^{s} \alpha_{s k}^{m}+\alpha_{j k}^{s} \alpha_{s i}^{m}+\alpha_{k i}^{s} \alpha_{s j}^{m}=0
$$

holds.

Recall that the inner product of two root vectors is minus one if and only if the corresponding entry of the Gram matrix is minus one. One consequence of this theorem is that the generalized Dynkin diagram $S(U)$ for a nilsoliton metric Lie algebra can not have exactly one dashed edge with weight minus one, or an equation like Equation (8) would need to be satisfied, but would not be able to hold. Another consequence is that if there are no minus one edges, the Jacobi condition holds automatically:

Corollary 14. Let $(\mathfrak{n}, Q)$ be an $n$-dimensional inner product space, and let $\mu$ be an element of $\Lambda^{2} \mathfrak{n}^{*} \otimes \mathfrak{n}$. Let $\mathcal{B}=\left\{X_{i}\right\}_{i=1}^{n}$ be an orthonormal basis for $(\mathfrak{n}, Q)$ and suppose that $\mu$ is $\mathcal{B}$-triangular. If the Gram matrix $U$ for $\left(\mathfrak{n}_{\mu}, Q\right)$ has no entries of -1 , then $\left(\mathfrak{n}_{\mu}, Q\right)$ is a Lie algebra.

Equation (8) may have continuous families of solutions, or more than one finite solution (because of sign choices in solving equations with squares). It is possible for different sign choices for the structure constants to give different nilpotent structures on an inner product space $\mathfrak{n}$, and hence nonisometric nilsolitons; i.e. there exists $\Lambda$ such that there are nonisometric $\mu$ in $\Omega_{\Lambda}$ with the same structure vector. This occurs in dimension six (see $\mu_{7}$ and $\mu_{8}$ in [Wil03]). Two such nilpotent structures have the same Ricci endomorphisms and derivations $D=\operatorname{Ric}-\beta \mathrm{Id}$, so there is not a one-to-one correspondence between nilsoliton metrics and eigenvalues of the derivation $D$. Now we prove Theorem 13 .

Proof. The Jacobi identity holds for basis vectors $X_{i}, X_{j}$, and $X_{k}$ if and only if

$$
\sum_{s=1}^{n} \alpha_{i j}^{s} \alpha_{s k}^{m}+\alpha_{j k}^{s} \alpha_{s i}^{m}+\alpha_{k i}^{s} \alpha_{s j}^{m}=0
$$


for all $m$. This equation holds for $X_{i}, X_{j}$ and $X_{k}$ by skew-symmetry if any index $i, j$ or $k$ is repeated, so the Jacobi condition holds if and only if Equation (9) is true for all $i, j, k$ and $m$ where $i, j$, and $k$ are distinct.

Now we will establish that when $i, j$ and $k$ are distinct, a product of the form $\alpha_{i j}^{l} \alpha_{l k}^{m}$ is nonzero if and only if $\left\langle Y_{i j}^{l}, Y_{l k}^{m}\right\rangle=-1$ for root vectors $Y_{i j}^{l}$ and $Y_{l k}^{m}$. Suppose that $\alpha_{i j}^{l} \alpha_{l k}^{m}$ is nonzero. As $\alpha_{i j}^{l} \neq 0$ and $\alpha_{l k}^{m} \neq 0$ and $\mu$ is $\mathcal{B}$-triangular by hypothesis, we have $i, j<l$ and $l, k<m$. It follows that $i<m$ and $j<m$. We also know that $l \neq k$ since $\alpha_{l k}^{m}$ is nonzero, and $i \neq k$ and $j \neq k$ because $i, j$, and $k$ were assumed to be distinct. The inner product of $Y_{i j}^{l}$ and $Y_{l k}^{m}$ is then

$$
\begin{aligned}
\left\langle Y_{i j}^{l}, Y_{l k}^{m}\right\rangle & =\left\langle\varepsilon_{i}^{T}+\varepsilon_{j}^{T}-\varepsilon_{k}^{T}, \varepsilon_{l}^{T}+\varepsilon_{k}^{T}-\varepsilon_{m}^{T}\right\rangle \\
& =\delta_{i l}+\delta_{i k}-\delta_{i m}+\delta_{j l}+\delta_{j k}-\delta_{j m}-\delta_{l l}-\delta_{l k}+\delta_{l m} \\
& =-1 .
\end{aligned}
$$

For the converse, suppose that the inner product of two root vectors is minus one. Then they must be of the form $Y_{i j}^{l}$ and $Y_{l k}^{m}$, or $Y_{i j}^{l}$ and $Y_{k l}^{m}$. In the first case, $(i, j, l)$ and $(l, k, m)$ are in $\Lambda\left(\mathfrak{n}_{\mu}, Q\right)$ so $\alpha_{i j}^{l} \alpha_{l k}^{m} \neq 0$, and in the second case, $(i, j, l)$ and $(k, l, m)$ are in $\Lambda\left(\mathfrak{n}_{\mu}, Q\right)$ so $\alpha_{i j}^{l} \alpha_{l k}^{m}=$ $-\alpha_{i j}^{l} \alpha_{k l}^{m} \neq 0$. We have proved the asserted equivalence in the first part of the theorem.

Now we suppose that $\left(\mathfrak{n}_{\mu}, Q\right)$ satisfies the Jacobi identity, so Equation (9) holds for all $i, j, k$ and $m$. Fix $m$ and suppose that the equation holds trivially. Then there are no nonzero terms of the form $\alpha_{i j}^{l} \alpha_{k l}^{m}$ so there are no root vectors whose inner product is minus one. If Equation (9) does not hold trivially for some value of $m$, we can simplify it slightly by noting that terms of the form $\alpha_{i j}^{s} \alpha_{k s}^{m}$ vanish when $s \geq m$ because $\mu$ is $\mathcal{B}$-triangular. This establishes the second assertion of the theorem.

q.e.d.

\section{Conditions Equivalent to the nilsoliton CONDition}

6.1. Derivations, gradings, and the nilsoliton condition. First we prove some theorems relating derivations, gradings, the nilsoliton condition, and eigenvalues. In the proof of Theorem 4.14 of [Heb98, Heber characterized derivations of a metric nilpotent algebra $(\mathfrak{n}, Q)$ in terms of vectors of the form $\varepsilon_{i}^{T}+\varepsilon_{j}^{T}-\varepsilon_{k}^{T}$ like the root vectors used in this paper. The next theorem is restatement and slight generalization of Heber's idea. For $\left(\mu_{1}, \ldots, \mu_{n}\right)^{T}$ in $\mathbb{R}^{n}$, define

$$
\begin{aligned}
\Sigma_{\left(\mu_{1}, \ldots, \mu_{n}\right)} & =\left\{(i, j, k) \in \Upsilon_{n} \mid \mu_{i}+\mu_{j}=\mu_{k}\right\} \\
& =\left\{(i, j, k) \in \Upsilon_{n} \mid Y_{i j}^{k}\left(\mu_{1}, \ldots, \mu_{n}\right)^{T}=0 .\right\}
\end{aligned}
$$


We say that a generalized Dynkin diagram $S(U)$ is a minor graph of another generalized Dynkin diagram $S\left(U^{\prime}\right)$ if the vertices of the first are a subset of the vertices of the second, and the generalized Cartan matrix for the first is the corresponding minor of the second.

Theorem 15. Let $(\mathfrak{n}, Q)$ be a n-dimensional nonabelian metric algebra with orthonormal basis $\mathcal{B}=\left\{X_{i}\right\}_{i=1}^{n}$. Let $D$ be the endomorphism of $\mathfrak{n}$ defined by $D\left(X_{i}\right)=\mu_{i} X_{i}$ for $1=1, \ldots, n$, and let $v_{D}=\left(\mu_{1}, \ldots, \mu_{n}\right)^{T}$.

The following properties are equivalent:

(1) $D$ is a derivation,

(2) $\Lambda(\mathfrak{n}, \mathcal{B})$ is a subset of $\Sigma_{\left(\mu_{1}, \ldots, \mu_{n}\right)}$,

(3) $v_{D}$ is in the kernel of the root matrix $Y_{\Lambda(\mathfrak{n}, \mathcal{B})}$ for $\Lambda(\mathfrak{n}, \mathcal{B})$.

(4) $S(\Lambda(\mathfrak{n}, \mathcal{B}))$ is a minor graph of $S\left(\Sigma_{\left(\mu_{1}, \ldots, \mu_{n}\right)}\right)$

Furthermore, if $\Lambda(\mathfrak{n}, \mathcal{B})$ is a subset of a set of integer triples $\Gamma \subset \Upsilon_{n}$, the kernel of the root matrix for $\Gamma$ is a subset of kernel of the root matrix for $\Lambda(\mathfrak{n}, \mathcal{B})$.

The theorem allows us to interpret the set $\Sigma_{\left(\mu_{1}, \ldots, \mu_{n}\right)}$ as the admissible nonzero structure constants for algebras admitting a derivation with eigenvalues $\mu_{1}, \ldots, \mu_{n}$.

Proof. Applying the definition of derivation to pairs of basis vectors, we see that $D$ is a derivation if and only if $\left[X_{i}, X_{j}\right]$ lies in the $\mu_{i}+\mu_{j}$ eigenspace for all basis vectors $X_{i}$ and $X_{j}$. Equivalently, since

$$
\left\langle D\left(\left[X_{i}, X_{j}\right]\right), X_{k}\right\rangle=\left\langle\sum_{l=1}^{n} \alpha_{i j}^{l} D\left(X_{l}\right), X_{k}\right\rangle=\alpha_{i j}^{k} \mu_{k},
$$

if $\alpha_{i j}^{k} \neq 0$, then $\mu_{k}=\mu_{i}+\mu_{j}$. Then $D$ is a derivation if and only if whenever the triple $(i, j, k)$ is in $\Lambda(\mathfrak{n}, \mathcal{B})$,

$$
0=\mu_{i}+\mu_{j}-\mu_{k}=Y_{i j}^{k}\left(\mu_{1}, \ldots, \mu_{n}\right)^{T}=Y_{i j}^{k} v_{D} ;
$$

or $\Lambda(\mathfrak{n}, \mathcal{B}) \subset \Sigma_{\left(\mu_{1}, \ldots, \mu_{n}\right)}$. This confirms the equivalence of the first and second properties.

Recall that because the rows of the root matrix $Y$ for a set of triples $\Gamma$ are the vectors $\left\{Y_{i j}^{k} \mid(i, j, k) \in \Gamma\right\}$, a vector $v_{D}$ is in the kernel of the root matrix for $\Gamma$ if and only if $Y_{i j}^{k} v_{D}=0$ for all $(i, j, k) \in \Gamma$. By this fact, the third property holds if and only if $Y_{i j}^{k} v_{D}=0$ for all $(i, j, k)$ in $\Lambda(\mathfrak{n}, \mathcal{B})$, which occurs if only if $\Lambda(\mathfrak{n}, \mathcal{B})$ is a subset of $\Sigma_{\left(\mu_{1}, \ldots, \mu_{n}\right)}$. Therefore the second and third properties are equivalent. Clearly the second and fourth properties are equivalent.

It is immediate from the same fact that whenever $\Lambda \subset \Gamma \subset \Upsilon_{n}$, the rows of the root matrix for $\Lambda$ are rows of the root matrix for $\Gamma$. If a vector $v$ is in the kernel of the root matrix for $\Gamma$, then $v$ is in 
the kernel of the root matrix for $\Lambda$. Letting $\Lambda=\Lambda(\mathfrak{n}, \mathcal{B})$ concludes the proof.

q.e.d.

It was shown in [Heb98] that for a a metric Lie algebra $(\mathfrak{n}, Q)$ there is a unique $\beta$ so that $\operatorname{Ric}-\beta \operatorname{Id}$ is a derivation, and it is

$$
\beta=-\frac{\operatorname{trace} D^{2}}{\operatorname{trace} D}=-\frac{\sum \mu_{i}^{2}}{\sum \mu_{i}} .
$$

If $v_{D}=\left(\mu_{1}, \ldots, \mu_{n}\right)$ is such a derivation, then we call $\left(\mu_{1}, \ldots, \mu_{n}\right)$ the eigenvalue type of $(\mathfrak{n}, Q)$. The following essential theorem translates the nilsoliton condition into a linear condition on the eigenvalues of the Ricci endomorphism. Theorem 1.6 of [Wol91] is an analogous theorem for Einstein solvmanifolds.

Theorem 16. Let $\left(\mathfrak{n}_{\mu}, Q\right)$ be a nonabelian metric algebra with Ricci eigenvector basis $\mathcal{B}$. Let $D=\operatorname{Ric}-\beta \mathrm{Id}$. The following are equivalent:

(1) $\left(\mathfrak{n}_{\mu}, Q\right)$ satisfies the nilsoliton condition with nilsoliton constant $\beta$

(2) The eigenvalue vector $v_{D}$ for $D$ with respect to $\mathcal{B}$ lies in the kernel of the root matrix for $(\mathfrak{n}, \mathcal{B})$ with respect to $\mathcal{B}$.

(3) For noncommuting eigenvectors $X$ and $Y$ for the nil-Ricci endomorphism with eigenvalues $\kappa_{X}$ and $\kappa_{Y}$, the bracket $[X, Y]$ is an eigenvector for the nil-Ricci endomorphism with eigenvalue $\kappa_{X}+\kappa_{Y}-\beta$.

(4) $Y_{i j}^{k}$ Ric $=\beta$ for all $(i, j, k)$ in $\Lambda\left(\mathfrak{n}_{\mu}, \mathcal{B}\right)$.

When $\left(\mathfrak{n}_{\mu}, Q\right)$ satisfies the soliton condition with nilsoliton constant $\beta$, the eigenspaces for the derivation $\mathrm{Ric}_{\mu}-\beta \mathrm{Id}$ are orthogonal.

Proof. As Ric and $D$ differ by a multiple of the identity, the basis $\mathcal{B}$ simultaneously diagonalizes the two maps Ric and $D$, with the $\kappa$ eigenspace for Ric equal to the $\kappa-\beta$ eigenspace for $D$. By definition, the metric Lie algebra $\left(\mathfrak{n}_{\mu}, Q\right)$ satisfies the nilsoliton condition with nilsoliton constant $\beta$ if and only if $D=\operatorname{Ric}-\beta$ Id is a derivation. The map $D$ is a derivation if and only if for noncommuting eigenvectors $X$ and $Y$ of $D$ with eigenvalues $\mu_{X}$ and $\mu_{Y}$, the bracket $[X, Y]$ is an eigenvector for the nil-Ricci endomorphism with eigenvalue $\mu_{X}+\mu_{Y}$. The $\mu$ eigenspace of $D$ is equal to the $\mu+\beta$ eigenspace of Ric; translating the equivalence into a statement about Ric eigenspaces shows the equivalence of the first and third properties.

By Theorem [15, $D$ is a derivation if and only if $v_{D}$ is in the kernel of $Y$. Hence the first two properties are equivalent. 
Assume $(i, j, k)$ is in $\Lambda\left(\mathfrak{n}_{\mu}, \mathcal{B}\right)$. By Theorem 8, $Y_{i j}^{k} \mathbf{R i c}=\kappa_{X_{i}}+\kappa_{X_{j}}-$ $\kappa_{X_{k}}$, hence $Y_{i j}^{k} \mathbf{R i c}=\beta$ if and only if $\kappa_{X_{k}}=\kappa_{X_{i}}+\kappa_{X_{j}}-\beta$. The equivalence of the third and fourth properties follows from writing arbitrary eigenvectors $X$ and $Y$ in terms of the basis eigenvectors.

The subspaces of the grading for $\left(\mathfrak{n}_{\mu}, Q\right)$ are orthogonal because they are eigenspaces of the nil-Ricci endomorphism. $\quad$ q.e.d.

J. Heber has shown that after rescaling, the eigenvalues for $D=$ Ric $-\beta$ Id for a nilsoliton $(\mathfrak{n}, Q)$ are positive integers with no common divisors (Theorem $\mathrm{C}$, [Heb98]). The previous theorem can be used in an elementary argument for why the nilsoliton constant $\beta$ for a nonabelian nilmanifold must be negative. Were it not, then by the previous theorem, the span of all Ricci eigenvectors with negative eigenvalues would be a subalgebra. But the Ricci endomorphism is negative semidefinite when restricted to the generating subspace $[\mathfrak{n}, \mathfrak{n}]^{\perp}$ by Theorem $[$, so then Ric would be negative definite on all of $\mathfrak{n}$. This is a contradiction, since again by Theorem 6, the Ricci endomorphism is positive definite on the center.

The next lemma is elementary.

Lemma 17. Let $\left(\mathfrak{n}_{\mu}, Q\right)$ be a metric algebra with orthonormal basis $\mathcal{B}=\left\{X_{i}\right\}_{i=1}^{n}$. Suppose $\mathfrak{n}_{\mu}$ has an $\mathbb{R}^{+}$-grading $\mathfrak{n}_{\mu_{i}}=\oplus_{\mu_{i} \in \mathbb{R}^{+}} \mathfrak{n}_{\mu}$, such that each basis vector $X_{i}$ is in some subspace $\mathfrak{n}_{\mu_{i}}$ for the grading. Then $\Lambda\left(\mathfrak{n}_{\mu}, \mathcal{B}\right) \subset \Sigma_{\left(\mu_{1}, \ldots, \mu_{n}\right)} \subset \Theta_{n}$

Proof. Since $\oplus_{\mu \in \mathbb{R}}+\mathfrak{n}_{\mu}$ is a grading, $\left[\mathfrak{n}_{\mu}, \mathfrak{n}_{\nu}\right]$ lies in $\mathfrak{n}_{\mu+\nu}$ for all $\mu$ and $\nu$ in $\mathbb{R}^{+}$. This directly implies that $\Lambda\left(\mathfrak{n}_{\mu}, \mathcal{B}\right) \subset \Sigma_{\left(\mu_{1}, \ldots, \mu_{n}\right)}$.

The space $\mathfrak{n}_{\mu+\nu}$ intersects both of the spaces $\mathfrak{n}_{\mu}$ and $\mathfrak{n}_{\nu}$ trivially as $\mu+$ $\nu$ is greater than both $\mu$ and $\nu$. The orthogonality of the eigenspaces and the compatibility of the basis with the grading imply that $\left\langle\left[X_{i}, X_{j}\right], X_{i}\right\rangle=$ 0 and $\left\langle\left[X_{i}, X_{j}\right], X_{j}\right\rangle=0$, so structure constants of the form $\alpha_{i j}^{i}$ are always zero, as claimed.

q.e.d.

The positivity of the eigenvalues for $D$ also gives the following useful corollary to the previous lemma.

Corollary 18. Let $\left(\mathfrak{n}_{\mu}, Q\right)$ be a nilsoliton metric algebra with Ricci eigenvector basis $\mathcal{B}$. Then $\mu$ is $\mathcal{B}$-triangular.

Proof. By definition, the Ricci eigenvector basis is orthonormal. Suppose that the nilsoliton constant for $(\mathfrak{n}, Q)$ is $\beta$ and let $D=\operatorname{Ric}-\beta$ Id . Then $\mathcal{B}$ is also an orthonormal basis of eigenvectors for $D$. The eigenvalues of $D$ are all positive, so $D$ defines an $\mathbb{R}^{+}$grading of $\mathfrak{n}$. q.e.d. 


\subsection{Proof of Theorem 1.}

Proof. Let $D=$ Ric $-\beta$ Id . The Ricci vector Ric is the eigenvalue vector for the nil-Ricci endomorphism with respect to a Ricci eigenvector basis. Let $v_{D}$ be the eigenvalue vector for $D$ with respect to $\mathcal{B}$. It is given by

$$
v_{D}=v_{\text {Ric }}-\beta v_{\text {Id }}=\mathbf{R i c}-\beta[1]_{n \times 1} .
$$

By Theorem 8, the Ricci vector equals $-\frac{1}{2} Y^{T}\left[\alpha^{2}\right]$, where $\left[\alpha^{2}\right]$ is the structure vector for $(\mathfrak{n}, Q)$ with respect to $\mathcal{B}$. Hence

$$
v_{D}=-\frac{1}{2} Y^{T}\left[\alpha^{2}\right]-\beta[1]_{n \times 1} \text {. }
$$

Multiplying both sides of the equation by the root matrix yields

$$
Y v_{D}=-\frac{1}{2} Y Y^{T}\left[\alpha^{2}\right]-\beta Y[1]_{n \times 1}
$$

For all triples $(i, j, k)$, we have $Y_{i j}^{k}[1]_{n \times 1}=[1]_{1 \times 1}$, so $Y[1]_{n \times 1}=[1]_{n \times 1}$. Making this substitution and noting that $Y Y^{T}=U$, we find that

$$
Y v_{D}=-\frac{1}{2} U\left[\alpha^{2}\right]-\beta[1]_{n \times 1} .
$$

Therefore, $Y v_{D}=0$ if and only if $U\left[\alpha^{2}\right]=-2 \beta[1]_{n \times 1}$. By Theorem [15, $Y v_{D}=0$ if and only if $D$ is a derivation. Thus, as asserted, $(\mathfrak{n}, Q)$ satisfies the nilsoliton condition with nilsoliton constant $\beta$ if and only if $U\left[\alpha^{2}\right]=-2 \beta[1]_{n \times 1}$.

q.e.d.

6.3. Examples. We next illustrate Theorem 1 and Theorem 13 with a continuation of Example 10.

Example 19. Let $(\mathfrak{n}, Q)$ be the metric nilpotent algebra with Ricci eigenvector basis $\mathcal{B}$ from Example 10. The set of root vectors $\left\{Y_{12}^{3}, Y_{13}^{4}, Y_{14}^{5}, Y_{23}^{5}\right\}$ is linearly independent, so the matrix $U$ is invertible. Thus, the system $U v=-2 \beta[1]_{4 \times 1}$ is consistent. The solution is

$$
\left[\begin{array}{l}
a^{2} \\
b^{2} \\
c^{2} \\
d^{2}
\end{array}\right]=-2 \beta\left[\begin{array}{llll}
3 & 0 & 1 & 0 \\
0 & 3 & 0 & 1 \\
1 & 0 & 3 & 1 \\
0 & 1 & 1 & 3
\end{array}\right]^{-1}\left[\begin{array}{l}
1 \\
1 \\
1 \\
1
\end{array}\right]=-\frac{2 \beta}{11}\left[\begin{array}{l}
3 \\
3 \\
2 \\
2
\end{array}\right] .
$$

We normalize $\beta$ to $-11 / 2$ and take positive roots, so $a=b=\sqrt{3}$ and $c=d=\sqrt{2}$ are structure constants defining a metric algebra satisfying the nilsoliton condition by Theorem 1. There are no entries of minus one in the Gram matrix, so by Corollary $13, \mathfrak{n}_{\mu}$ satisfies the Jacobi identity and is a Lie algebra. Up to rescaling and metric isomorphism, this is the unique nilsoliton metric in the equivalence class $\Omega_{\Lambda} \subset \mathcal{R}(\mathfrak{n}, \mathcal{B})$. The derivation $D=\operatorname{Ric}_{\mu}-\beta$ Id has eigenvalue vector

$$
\left(\mu_{1}, \mu_{2}, \mu_{3}, \mu_{4}, \mu_{5}\right)^{T}=\mathbf{R i c}_{\mu}^{\mathcal{B}}+\frac{11}{2}[1]_{5 \times 1}=\frac{3}{2}(1,2,3,4,5)^{T} .
$$


THE EXISTENCE OF SOLITON METRICS FOR NILPOTENT LIE GROUPS 25

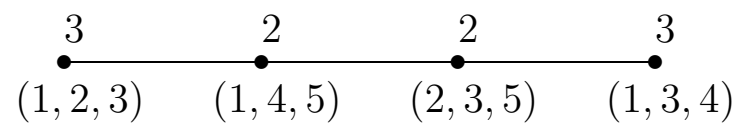

Figure 1. The weighted generalized Dynkin diagram $S(U)$ for Example 12

The derivation $D$ defines a metric solvable extension of $\left(\mathfrak{n}_{\mu}, Q\right)$ which is Einstein.

The Dynkin diagram $S(U)$, illustrated in Figure 1, is that of $A_{4}$ and the structure vector defines a weighting so that vertices have weights $3,2,2$, and 3 as shown. It is easy to check that Equation (2) holds for the weighting of Dynkin diagram- triple the weight of any vertex plus the sum of the weights of the adjacent vertices has the constant value of $11=-2 \beta$. There are no $(-1)$-weighted edges due to the lack of -1 s in the Gram matrix; this tells us that the Jacobi condition holds.

The next example illustrates both Theorem 1 and Theorem 13 .

Example 20. Let $\mathfrak{n}$ be a six-dimensional vector space equipped with the inner product $Q$ with respect to which $\mathcal{B}=\left\{X_{i}\right\}_{i=1}^{6}$ is an orthonormal basis. Consider the level set $\Omega_{\Lambda}$ in $\Lambda^{2} \mathfrak{n}^{*} \otimes \mathfrak{n}$ for

$$
\Lambda=\{(1,2,4),(1,4,5),(1,5,6),(2,3,5),(3,4,6)\}
$$

The Gram matrix of $\Lambda\left(\mathfrak{n}_{\mu}, \mathcal{B}\right)$ for $\mu$ in $\Lambda$ is $U=\left[\begin{array}{ccccc}3 & 0 & 1 & 1 & -1 \\ 0 & 3 & 0 & 1 & 1 \\ 1 & 0 & 3 & -1 & 1 \\ 1 & 1 & -1 & 3 & 1 \\ -1 & 1 & 1 & 1 & 3\end{array}\right]$.

A computation shows that a structure vector $\left[\alpha^{2}\right]$ is a solution to $U v=[1]_{5 \times 1}$ if and only if it is of the form

$$
\left[\alpha^{2}\right]=\left[\begin{array}{c}
\left(\alpha_{12}^{4}\right)^{2} \\
\left(\alpha_{14}^{5}\right)^{2} \\
\left(\alpha_{15}^{6}\right)^{2} \\
\left(\alpha_{23}^{5}\right)^{2} \\
\left(\alpha_{34}^{6}\right)^{2}
\end{array}\right]=\frac{1}{20}\left[\begin{array}{l}
1 \\
4 \\
9 \\
8 \\
0
\end{array}\right]+\frac{s}{20}\left[\begin{array}{c}
1 \\
0 \\
-1 \\
-1 \\
1
\end{array}\right]
$$

The only entries of minus one in the Gram matrix are those from the inner products $\left\langle Y_{12}^{4}, Y_{34}^{6}\right\rangle$ and $\left\langle Y_{15}^{6}, Y_{23}^{5}\right\rangle$, which correspond to nontrivial 
terms

$$
\begin{aligned}
& \left(\alpha_{15}^{6} \alpha_{23}^{5}\right)^{2}=\frac{1}{20^{2}}(9-s)(8-s) \quad \text { and } \\
& \left(\alpha_{12}^{4} \alpha_{34}^{6}\right)^{2}=\frac{1}{20^{2}}(1+s) s
\end{aligned}
$$

in the Jacobi identity in Equation (9). Both of the expressions are from the nontriviality of the equation with $\{i, j, k\}=\{1,2,3\}$ and $m=6$. By Theorem 13, the Jacobi identity holds for $\left(\mathfrak{n}_{\mu}, \mathcal{B}\right)$ with structure vector $\left[\alpha^{2}\right]$ if and only if

$$
\sum_{s<6} \alpha_{12}^{s} \alpha_{s 3}^{6}+\alpha_{23}^{s} \alpha_{51}^{6}+\alpha_{31}^{s} \alpha_{s 2}^{6}=0 .
$$

Substituting in for the other structure constants (all zero), we find that

$$
\begin{aligned}
\alpha_{12}^{4} \alpha_{34}^{6}+\alpha_{23}^{4} \alpha_{15}^{6} & =0 \\
\left(\alpha_{12}^{4}\right)^{2}\left(\alpha_{34}^{6}\right)^{2} & =\left(\alpha_{23}^{4}\right)^{2}\left(\alpha_{15}^{6}\right)^{2} \\
(9-s)(8-s) & =(1+s) s \\
s & =4 .
\end{aligned}
$$

When we let $s=4$ in Equation (10), we get

$$
\left[\alpha^{2}\right]=\frac{1}{20}(5,4,4,5,4)^{T}
$$

and the resulting $\left(\mathfrak{n}_{\mu}, Q\right)$ is a Lie algebra. After rescaling and solving for structure constants from $\left[\alpha^{2}\right]$, we find that

$$
\begin{gathered}
{\left[X_{1}, X_{2}\right]=\sqrt{5} X_{4} \quad\left[X_{1}, X_{4}\right]=2 X_{5} \quad\left[X_{1}, X_{5}\right]=2 X_{6}} \\
{\left[X_{2}, X_{3}\right]=-\sqrt{5} X_{5} \quad\left[X_{3}, X_{4}\right]=2 X_{6}}
\end{gathered}
$$

defines a nilsoliton metric Lie algebra. We could have made other sign choices for the structure constants as long as the signs of $\alpha_{12}^{4} \alpha_{34}^{6}$ and $\alpha_{23}^{4} \alpha_{15}^{6}$ were different; however, all these sign choices yield isometric nilsolitons.

6.4. Applications to graded metric filiform Lie algebras. In this section, we restate Theorem 15 of Section 6 in terms of the generalized Dynkin diagrams and give an application to filiform nilsoliton metric Lie algebras.

Suppose that an $n$-dimensional metric nilpotent Lie algebra $(\mathfrak{n}, Q)$ admits a symmetric derivation $D$ with eigenvalues $\mu_{1}, \ldots, \mu_{n}$. By Lemma 2.2 of [Heb98], $D$ commutes with the Ricci endomorphism of $(\mathfrak{n}, Q)$, so there exists a Ricci eigenvector basis $\mathcal{B}=\left\{X_{i}\right\}_{i=1}^{n}$ adapted to $D$; that is, each $X_{i}$ is an eigenvector for $D$. By Theorem $[15, \Lambda(\mathfrak{n}, \mathcal{B})$ is a subset 
of $\Sigma_{\left(\mu_{1}, \ldots, \mu_{n}\right)}$. Conversely, if $(\mathfrak{n}, Q)$ has Ricci eigenvector basis $\mathcal{B}$ and $\Lambda(\mathfrak{n}, \mathcal{B})$ is a subset of $\Sigma_{\left(\mu_{1}, \ldots, \mu_{n}\right)}$, then by Theorem 16$]\left(X_{i}\right)=\mu_{i} X_{i}$ defines a derivation of $\mathfrak{n}$.

The following theorem summarizes the previous discussion.

Theorem 21. Let $(\mathfrak{n}, Q)$ be a n-dimensional metric nilpotent Lie algebra with Ricci eigenvector basis $\mathcal{B}$. Suppose that $D$ is a symmetric derivation of $\mathfrak{n}$ with eigenvalue vector $v_{D}=\left(\mu_{1}, \ldots, \mu_{n}\right)$. Then the generalized Dynkin diagram for $\Lambda(\mathfrak{n}, \mathcal{B})$ is a minor graph of the generalized Dynkin diagram of $\Sigma_{\mu_{1}, \ldots, \mu_{n}}$, which in turn is a subset of $\Theta_{n}$. Conversely, if $\Lambda(\mathfrak{n}, \mathcal{B})$ is a minor graph graph of $\Sigma_{\mu_{1}, \ldots, \mu_{N}}$ then the endomorphism $D$ of $\mathfrak{n}$ defined by $D\left(X_{i}\right)=\mu_{i} X_{i}$ for $i=1, \ldots, n$ is a symmetric derivation $D$ of $\mathfrak{n}$.

For example, the characteristically nilpotent Lie algebra defined by Dixmier and Lister in Example 24 does not admit semisimple derivation, so its generalized Dynkin diagram $S(\Lambda)$ can not be a minor graph of $S\left(\Sigma_{\left(\mu_{1}, \ldots, \mu_{n}\right)}\right)$ for any $\left(\mu_{1}, \ldots, \mu_{n}\right)$ in $\mathbb{R}^{n}$.

The study of the graphs $\Sigma_{\left(\mu_{1}, \ldots, \mu_{n}\right)}$ and $\Theta_{n}$ has two applications to nilsolitons. First, one can show that a generalized Dynkin diagram $S(U)$ is realized as the generalized Dynkin diagram for a nilsoliton metric Lie algebras by finding $S(U)$ as a minor graph of some $S\left(\Sigma_{\left(\mu_{1}, \ldots, \mu_{n}\right)}\right)$, finding a nilsoliton weighting on $S(U)$ by solving $U v=[1]$, defining an algebra using structure constants from the weighting, and checking the Jacobi identity with Theorem 13. Second, one can look for graph-theoretic or combinatorial obstructions for a metric nilpotent Lie algebra to admit a symmetric derivation by finding properties of the graphs $S\left(\Sigma_{\left(\mu_{1}, \ldots, \mu_{n}\right)}\right)$ that give restrictions on the types of minor graphs they can have.

In the next example we describe the generalized Dynkin diagrams for the sets $\Sigma_{n}:=\Sigma_{(1,2, \ldots, n)}$ with multiplicities zero or one.

Example 22. Let $(\mathfrak{n}, Q)$ be an inner product space of dimension $n$ with orthonormal basis $\mathcal{B}=\left\{X_{i}\right\}_{i=1}^{n}$. Let $\Sigma_{n}$ be the set of integer triples defined by

$$
\begin{aligned}
\Sigma_{n} & =\Sigma_{(1,2, \ldots, n)} \\
& =\{(i, j, i+j) \mid 1 \leq i<j \leq n, i+j \leq n\} .
\end{aligned}
$$

Let $U_{\Sigma_{n}}$ denote the Gram matrix for $\Sigma_{n}$ and let $S\left(\Sigma_{n}\right)=S\left(U_{\Sigma_{n}}\right)$ denote the generalized Dynkin diagram associated to $U_{\Sigma_{n}}$.

It is easy to check that $S\left(\Sigma_{3}\right)$ is the Dynkin diagram of type $A_{1}$, $S\left(\Sigma_{4}\right)$ is the Dynkin diagram of type $A_{1} \oplus A_{1}$, and $S\left(\Sigma_{5}\right)$ is the Dynkin diagram of type $A_{4}$. When $n \geq 6$, the Gram matrix always has entries of minus one coming from triples of the form $(i, j, k)$ and $(l, k, m)$ with $l \neq, i, l \neq j$ in $\Sigma_{n}$ such as $(1,3,4)$ and $(2,4,6)$ in $\Sigma_{6}$. 


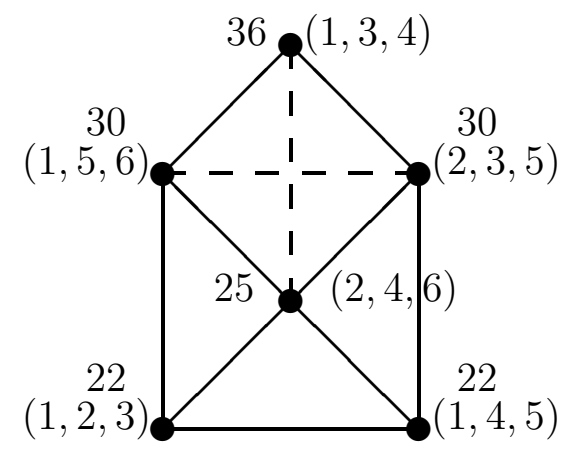

Figure 2. The generalized Dynkin diagram $S(U)$ for $\Sigma_{6}$ with a nilsoliton weighting

The generalized Dynkin diagram for $\Sigma_{6}$ is in Figure 2. It was shown in $\mathrm{McD}$ that $\Delta_{6}$ admits a one-parameter family of nilsoliton weightings with $\beta=-2$, and the Jacobi condition from the two dotted edges as described by Theorem 13 gives a constraint so that up to scaling, there is a unique nilsoliton metric $(\mathfrak{n}, Q)$ with $\Lambda(\mathfrak{n}, \mathcal{B})=\Sigma_{6}$ for a Ricci eigenvector basis $\mathcal{B}$.

\section{The solution SPACE To $U v=[1]$}

There are four distinct possibilities for the solution space of a linear system $U v=-2 \beta[1]_{m \times 1}$ arising from Theorem 11. Solution spaces of the first four of the possible types occur: the first type in Example 10. the second types and third types from horospheres for a quaternionic hyperbolic space and the Cayley plane in Examples 27 and 28, and the fourth type in Example 24. As symmetric Cartan matrices of finite and affine type have been classified, it is not hard to list all systems of finite and affine type and to find solutions to $A v=\mu[1]$ in each case. See page 54 of [Kac90] for the solutions in the affine case, presented as weighted Dynkin diagrams.

Proof of Theorem 2. Substituting $U=c A+d[1]_{m \times m}$ into $U v=\lambda[1]_{m \times 1}$ and using the fact that $[1]_{m \times m} v=\left([1]_{m \times 1} \cdot v\right)[1]_{m \times 1}$, we derive the equivalent equation

$$
A v=\frac{\lambda-d\left([1]_{m \times 1} \cdot v\right)}{c}[1]_{m \times 1} .
$$

Viewing $\lambda$ and $\mu$ as parameters, the two families of linear equations $U v=\lambda[1]_{m \times 1}$ and $A v=\mu[1]_{m \times 1}$ have solutions that are in bijective correspondence: if $v$ is a solution to $U v=\lambda[1]_{m \times 1}$, then $v$ is a solution 
to $A v=\mu[1]_{m \times 1}$ with parameter

$$
\mu=M(\lambda, v)=\frac{\lambda-d\left([1]_{m \times 1} \cdot v\right)}{c}
$$

and if $v$ is a solution to $A v=\mu[1]_{m \times 1}$, then $v$ is a solution to $U v=$ $\lambda[1]_{m \times 1}$ with parameter

$$
\lambda=L(\mu, v)=c \mu+d\left([1]_{m \times 1} \cdot v\right) .
$$

If $U v=\lambda[1]_{m \times 1}$, then $L(M(\lambda, v), v)=\lambda$ and if $A v=\mu[1]_{m \times 1}$, then $M(L(\mu, v), v)=\mu$.

By Theorem 5, there are three mutually exclusive cases for the generalized Cartan matrix $A$ : it is of finite type, affine type, or indefinite type. We consider each case in turn.

First suppose that $A$ is of finite type. By Theorem 5, $A$ is invertible, so the equation $A v=[1]_{m \times 1}$ has a unique solution $v_{0}$, and as $A v_{0}=$ $[1]_{m \times 1}$ is positive, $v_{0}$ is positive. As the vector $v_{0}$ is a solution to $A v=\mu[1]_{m \times 1}$ with $\mu=1$, it is a solution to $U v=\lambda[1]_{m \times 1}$ with $\lambda=L\left(1, v_{0}\right)=c+d\left([1]_{m \times 1} \cdot v_{0}\right)$. The positivity of $c$ and $d$ and the fact that $v_{0}>0$ force $L\left(1, v_{0}\right)$ to be positive. Rescaling gives a solution $-\frac{2 \beta}{L\left(1, v_{0}\right)} v_{0}$ to $U v=-2 \beta[1]_{m \times 1}$. By hypotheses $\beta<0$, so $-\frac{2 \beta}{L\left(1, v_{0}\right)} v_{0}$ is a positive vector. It is the unique solution to $U v=-2 \beta[1]_{m \times 1}$; were it not unique, there would exist a vector $v$ in the solution space, not a multiple of $v_{0}$, and sufficiently close to $v_{0}$ that $M(-2 \beta, v) \neq 0$ $\left(M\left(-2 \beta, v_{0}\right)=1\right)$. Then $\frac{1}{M(-2 \beta, v)} v$ would be a second solution to $A v=[1]_{m \times 1}$, contradicting the nonsingularity of $A$.

Suppose that $A$ is of affine type. By Theorem [5, there exists positive $v_{0}$ with $A v_{0}=0$. Then $v_{0}$ is a solution to $A v=\mu[1]_{m \times 1}$ with $\mu=0$, making $v_{0}$ a solution to $U v=\lambda[1]_{m \times 1}$ with $\lambda=L\left(0, v_{0}\right)=$ $d\left([1]_{m \times 1} \cdot v\right)>0$. Rescaling to $v=-\frac{2 c v \beta}{L\left(0, v_{0}\right)} v_{0}$ gives a positive solution $v$ to Equation $U v=-2 \beta[1]_{m \times 1}$. Now we show that the solution $v_{0}$ is unique. Suppose that $v_{1}$ is another solution to $U v=-2 \beta[1]_{m \times 1}$. Then $v_{1}$ is a solution to $A v=\mu[1]_{m \times 1}$ with $\mu=M\left(-2 \beta, v_{1}\right)$. If $\mu \geq 0$, then $A v_{1} \geq 0$, and Theorem 5 implies that actually $A v_{1}=0$. If $\mu \leq 0$; then $A\left(-v_{1}\right) \geq 0$, by again by Theorem [5, $A\left(-v_{1}\right)=0$. Thus $v_{1}$ lies in the one-dimensional kernel of $A$ and must be a multiple $k$ of $v_{0}$. Then $U\left(v_{0}\right)=-2 \beta[1]_{m \times 1}=U\left(k v_{0}\right)$ and the nonzero vector $U\left(v_{0}\right)$ is a multiple $k$ of itself. Hence $k=1$ and $v_{0}=v_{1}$.

Now suppose that $A$ is of indefinite type. We must show that if $U v=$ $-2 \beta[1]_{m \times 1}$ is consistent and there exists a positive solution, then Case (Ind1) holds. First we assume that $U v=-2 \beta[1]_{m \times 1}$ is consistent, and we show that $\operatorname{ker} U \subset \operatorname{ker} A$. Recall that a matrix equation $B x=y$ is consistent if and only if $y$ is in range $B=\left(\operatorname{ker} B^{T}\right)^{\perp}$, so $\operatorname{ker} U \subset[1]_{m \times 1}^{\perp}$. 
Suppose that $v \in \operatorname{ker} U$. Then $v$ is a solution to $U v=\lambda[1]_{m \times 1}$ with $\lambda=0$. The one-to-one correspondence between solution spaces makes $v_{0}$ a solution to $A v=\mu[1]_{m \times 1}$ with $\mu=M\left(0, v_{0}\right)=0$. Thus, $v \in \operatorname{ker} A$.

Let $v_{0}$ be a fixed positive solution to $U v=-2 \beta[1]_{m \times 1}$. The bijective correspondence of solution spaces makes $v_{0}$ a solution to $A v=\mu[1]_{m \times 1}$ with $\mu=M\left(-2 \beta, v_{0}\right)$. The complete solution space to $A v=\mu[1]_{m \times 1}$ with $\mu=M\left(-2 \beta, v_{0}\right)$ is $\left\{v_{0}+w \mid w \in \operatorname{ker} A\right\}$.

Any vector $v_{0}+w$ in this set is a solution to $U v=\lambda[1]_{m \times 1}$ with $\lambda=L\left(M\left(-2 \beta, v_{0}\right), v_{0}+w\right)$. Since $L\left(M\left(-2 \beta, v_{0}\right), v_{0}\right)=-2 \beta>0$, by continuity, for $w$ in a small neighborhood $U$ of the zero vector in $\operatorname{ker} A$, $M\left(L\left(-2 \beta, v_{0}\right), v_{0}+w\right)>0$. Rescaling, we find that the solution space to the system $U v=-2 \beta[1]_{m \times 1}$ contains

$$
\left\{\frac{-2 \beta}{L\left(M\left(-2 \beta, v_{0}\right), v_{0}+w\right)}\left(v_{0}+w\right) \mid w \in U\right\} .
$$

This is a space of the same dimension as $\operatorname{ker} A$. Hence, $\operatorname{dim} \operatorname{ker} A \leq$ $\operatorname{dim} \operatorname{ker} U$, so $\operatorname{ker} A=\operatorname{ker} U$.

In the indefinite case, the fact that ker $A \subset[1]_{m \times 1}^{\perp}$ implies that the set of nonnegative solutions is bounded and forms a simplex of dimension $\operatorname{ker} A$.

Now assume that $U$ is the Gram matrix for a metric nilpotent Lie algebra $(\mathfrak{n}, Q)$ with respect to some basis $\mathcal{B}$. Let $Y$ be the $m \times n$ root matrix for $\Lambda(\mathfrak{n}, \mathcal{B})$. By definition, $U=Y^{T} Y$. We will show that the kernel of the symmetric matrix $U$ is contained in $[1]_{m \times 1}^{\perp}$. Suppose that $v$ is in the kernel of $U$. The kernel of $U$ is equal to the kernel of $Y^{T}$; hence $v$ is in the kernel of $Y^{T}$. Then

$$
[1]_{1 \times n} Y^{T} v=[1]_{1 \times n}[0]_{n \times 1}=[0]_{1 \times 1} .
$$

By definition, the rows of $Y$ are the structure vectors $Y_{i j}^{k}=\varepsilon_{i}^{T}+\varepsilon_{j}^{T}-\varepsilon_{k}^{T}$ for $(i, j, k)$ in $\Lambda$. The product of the row vector $[1]_{1 \times n}$ and any column of $Y^{T}$ is one, so $[1]_{1 \times n} Y^{T}=[1]_{1 \times m}$. Substituting, we conclude that $[1]_{1 \times m} v=[0]_{1 \times 1}$ as desired.

q.e.d.

\section{All SOliton Metrics in A ClASs $\Omega_{\Lambda}$}

Here is the proof of Theorem 3 .

Proof. Fix a subset $\Lambda$ of $\Theta_{n}$ and the root matrix $Y$ and Gram matrix $U$ for $\Lambda$. As $\Lambda \subset \Theta_{n}$, the matrix $A=2 U-4[1]_{m \times m}$ is a generalized Cartan matrix by Theorem 12 .

Suppose that $U$ is indecomposable. Theorem 2 then applies with $U=\frac{1}{2} A+2[1]_{m \times m}$. In Cases (Fin) and (Aff) of the theorem, there is a unique positive solution $\left[\alpha^{2}\right]$ to $U v=-2 \beta[1]_{m \times 1}$. There are $2^{m}$ different 
$\mu$ with this structure vector, one for each choice of sign in each entry of $\left[\alpha^{2}\right]$. If we are in Case (Ind1) of Theorem 2, $U$ is of indefinite type and there exists a solution $v_{0}$ to $U v=[1]_{m \times 1}$ with positive entries. The set of solutions to $U v=[1]_{m \times 1}$ in the cone $\left\{v \in \mathbb{R}^{m} \mid v \geq 0\right\}$ is a simplex of the same dimension as ker $A$. Taking square roots entry-wise and allowing all sign choices gives $2^{m}$ simplices of $\mu$ so that $\left(\mathfrak{n}_{\mu}, Q\right)$ satisfies $U\left[\alpha^{2}\right]=-2 \beta[1]_{m \times 1}$. Only the interiors of these simplices lie inside $\Omega_{\Lambda}$. In Case (Ind3) of Theorem 2 there are no $\mu$ in $\Omega_{\Lambda}$ whose structure vector is a solution to $U v=-2 \beta[1]_{m \times 1}$.

By Equation (5), if $\left(\mathfrak{n}_{\mu}, Q\right)$ has structure vector $v_{0}+v$, with $v$ in $\operatorname{ker} A=\operatorname{ker} Y^{T}$, then the Ricci vector is

$$
\operatorname{Ric}_{\mu}^{\mathcal{B}}=-\frac{1}{2} Y^{T}\left(v_{0}+v\right)=-\frac{1}{2} Y^{T} v_{0} .
$$

In other words, for all metric algebras $\left(\mathfrak{n}_{\mu}, Q\right)$ with structure vector $v_{0}+v$ that satisfy the nilsoliton condition, the Ricci vector is the same. Since $\mathcal{B}$ is a common Ricci eigenvector basis for all such $\left(\mathfrak{n}_{\mu}, Q\right)$, once the Ricci vector is constant, all the nil-Ricci endomorphisms agree.

If $U$ is decomposable, after permuting the basis vectors, it can be written as the block diagonal sum of indecomposable Gram matrices $U_{1}, U_{2}, \ldots, U_{r}$. With respect to the new basis, the vector $[1]_{m \times 1}$ is still written as $[1]_{m \times 1}$. The solution space to $U v=[1]_{m \times 1}$ is the product of the solution spaces to the systems $U_{i} v=[1]_{m_{i} \times 1}$, where $U_{i}$ is $m_{i} \times m_{i}$ and $i=1, \ldots, r$. If $U$ is nonsingular, then each $U_{i}$ is nonsingular with unique solution $v_{i}$ to $U_{i} v=[1]_{m_{i} \times 1}$, making $v=v_{1} \times \ldots \times v_{r}$ the unique solution to $U v=[1]_{m \times 1}$. If $U$ is nonsingular and there is some $i$ so that $U_{i} v=[1]_{m_{i} \times 1}$ has no solution with positive entries, then $U v=[1]_{m \times 1}$ has no solution with positive entries. If $U$ is nonsingular and $U_{i} v=[1]_{m_{i} \times 1}$ has positive solutions for all $i$, then the solution space is the nontrivial product of the solution spaces to the equations $U_{i} v=[1]_{m_{i} \times 1}$. The product of simplices is a simplex and the dimension of a product is the sum of the dimensions of the factors, so the theorem holds for the decomposable case. When $U$ is decomposable, and $(\mathfrak{n}, Q)$ is nilsoliton with nilsoliton constant $\beta$, then $\left(\mathfrak{n}_{i}, Q_{i}\right)$ is also nilsoliton with nilsoliton constant $\beta$, where $\mathfrak{n}_{i}$ is the subspace for the submatrix $U_{i}$ and $Q_{i}$ is the restriction of $Q$ to that subspace. The Ricci vector for $(\mathfrak{n}, Q)$ is obtained by concatenating the Ricci vectors $\mathbf{R i c}_{i}$ for $\left(\mathfrak{n}_{i}, Q_{i}\right)$. Thus, the constancy of the Ricci vector for nilsoliton metrics in the same equivalence class holds in the decomposable case also. q.e.d.

As a corollary to Theorem [3, we find that any time the number of nontrivial structure constants $\alpha_{i j}^{k}$ with $i<j$ exceeds the dimension 
of a nilsoliton $\left(\mathfrak{n}_{\mu}, Q\right)$, there are continuous families of metric algebras around $\left(\mathfrak{n}_{\mu}, Q\right)$ also satisfying the nilsoliton condition. The Jacobi identity may prohibit members of this continuous family from being nilsoliton metric Lie algebras.

Corollary 23. Let $(\mathfrak{n}, Q)$ an $n$-dimensional inner product space and let $\mathcal{B}$ be a orthonormal basis for $\mathfrak{n}$. Let $\Omega_{\Lambda}$ be a nontrivial level set for $\mu \mapsto \Lambda$ so that $\Lambda \subset \Theta_{n}$. and $\|\Lambda\|>n$. Let $\beta<0$. Then the Gram matrix $U$ for $\Omega$ is singular, and either $\Omega_{\Lambda}$ contains no $\mu$ satisfying $U v=-\beta[1]_{m \times 1}$ or there is a continuous family of dimension at least $m-n$ of $\mu$ in $\Omega_{\Lambda}$ so that $\left(\mathfrak{n}_{\mu}, Q\right)$ satisfies $U v=-\beta[1]_{m \times 1}$.

Proof. The rank of $U$ is equal to the number of independent root vectors. The root vectors are $1 \times n$ in size, so the maximal rank of $U$ is $n$. By Theorem 3 , the set of $\mu$ in $\Omega_{\Lambda}$ that satisfy $U\left[\alpha^{2}\right]=-2 \beta[1]_{m \times 1}$ is either empty or of dimension $\operatorname{ker} U=m-\operatorname{rank} U \geq m-n$, where $m=\|\Lambda\|$.

q.e.d.

In Example 28 we will have $(m, n)=(28,15)$ and an eight-parameter subfamily of solutions. The nilpotent metric Lie algebra $(\mathfrak{n}, Q)$ for quaternionic space $H^{n} \mathbb{H}$ of dimension $4 n$ has $(m, n)=(6 n, 4 n+3)$ in its most natural representation (as in Example 27), therefore for $n \geq 2$ there will be continuous families of nilsolitons around $(\mathfrak{n}, Q)$ for $n \geq 2$. In Example 27, we will have $(m, n)=(12,11)$ and a two-parameter subfamily of solutions. A similar computation for $H^{3}(\mathbb{H})$ not included here yields a four-parameter family of Einstein solvmanifolds around quaternionic hyperbolic space of dimension sixteen.

\section{EXAMPles}

9.1. A characteristically nilpotent example. The next example is of Case (Ind2) in Theorem 2, when solutions to $U v=[1]$ exist, but the solution vectors never have all entries positive. The example, due to J. Dixmier and W. G. Lister, was the first example of a nilpotent Lie algebra with a nilpotent derivation algebra. A Lie algebra $\mathfrak{g}$ so that the Lie algebra of derivations of $\mathfrak{g}$ is nilpotent is called characteristically nilpotent (see [Kha02] for a survey). Such a Lie algebra admits no semisimple derivations, and hence can not admit a nilsoliton metric. All nilpotent structures of dimension six or less admit nilsoliton metrics (see Lau02] and Wil03] for a classification), and the associated derivation Ric $-\beta$ Id is nontrivial and semisimple. The lowest dimension in which characteristically nilpotent Lie algebras occur is ([Fav72]).

Example 24. ([DL57]) Let $(\mathfrak{n}, Q)$ be the metric Lie algebra with orthonormal basis $\mathcal{B}=\left\{X_{i}\right\}_{i=1}^{8}$ and nontrivial structure constants encoded 
THE EXISTENCE OF SOLITON METRICS FOR NILPOTENT LIE GROUPS 33

in the Lie brackets

$$
\begin{array}{lll}
{\left[X_{1}, X_{2}\right]=X_{5}} & {\left[X_{1}, X_{3}\right]=X_{6}} & {\left[X_{1}, X_{4}\right]=X_{7}} \\
{\left[X_{1}, X_{5}\right]=-X_{8}} & {\left[X_{2}, X_{3}\right]=X_{8}} & {\left[X_{2}, X_{4}\right]=X_{6}} \\
{\left[X_{2}, X_{6}\right]=-X_{7}} & {\left[X_{3}, X_{4}\right]=-X_{5}} & {\left[X_{3}, X_{5}\right]=-X_{7}} \\
& {\left[X_{4}, X_{6}\right]=-X_{8}} &
\end{array}
$$

By Corollary 7 , the basis $\mathcal{B}$ is a Ricci eigenvector basis. We find using MAPLE that $U$ is singular with the vectors

$$
\begin{aligned}
& v_{1}=(1,-1,0,0,0,0,-1,0,1,0)^{T} \quad \text { and } \\
& v_{2}=(0,1,0,-1,0,0,0,-1,0,1)^{T}
\end{aligned}
$$

spanning $\operatorname{ker} U=\operatorname{ker} A=\operatorname{ker} Y^{T}$. The general solution to $U v=[1]_{10 \times 1}$ is

$$
v=\left(-\frac{1}{2}, \frac{5}{2},-7,6,-7, \frac{5}{2}, 6, \frac{11}{2}, 0,0\right)^{T}+s v_{1}+t v_{2}
$$

none of these solutions satisfy $v>0$, as the third coordinate is always negative, so $A$ is of indefinite type by Theorem 5. Note that $v_{1}$ and $v_{2}$ are orthogonal to $[1]_{10 \times 1}$ since $U v=[1]_{10 \times 1}$ and $A v=[1]_{10 \times 1}$ are consistent.

9.2. The class $L_{n}$ of filiform Lie algebras. Let $\mathfrak{g}$ be a Lie algebra. The descending central series of $\mathfrak{g}$ is defined by $\mathfrak{g}^{(1)}=\mathfrak{g}$ and $\mathfrak{g}^{(j+1)}=$ $\left[\mathfrak{g}, \mathfrak{g}^{(j)}\right]$ for $j>1$. The Lie algebra $\mathfrak{g}$ is nilpotent if and only if there is an integer $k$ so that $\mathfrak{g}^{(k)}$ is trivial. If $k$ is the smallest integer so that $\mathfrak{g}^{(k+1)}$ is trivial, then $\mathfrak{g}$ is said to be $k$-step nilpotent.

A $k$-step nilpotent Lie algebra of dimension $n$ is called filiform if $k+1=n$. See [Kha02] for a survey of filiform and characteristically nilpotent Lie algebras. The $(n+1)$-dimensional filiform nilpotent Lie algebra $L_{n}$ is defined with respect to the basis $\left\{X_{i}\right\}_{i=0}^{n}$ by the bracket relations

$$
\left[X_{0}, X_{i}\right]=X_{i+1} \quad \text { for } i=1, \ldots, n-1 .
$$

J. Lauret has shown using variational methods that every Lie algebra in this family admits an inner product $Q$ so that $\left(L_{n}, Q\right)$ is a nilsoliton metric Lie algebra. We give an alternate algebraic proof of this fact.

Theorem 25 ([Lau02]). For all $n$, the filiform nilpotent Lie algebra $L_{n}$ admits a unique nilsoliton metric.

Proof. Take the inner product $Q$ on the vector space $\mathfrak{n}=\operatorname{span}\left\{X_{i}\right\}_{i=0}^{n}$ with respect to which $\mathcal{B}=\left\{X_{i}\right\}_{i=0}^{n}$ is orthonormal. We want to find nonzero values of $a_{i}$ such that the bracket relations

$$
\left[X_{0}, X_{i}\right]=a_{i} X_{i+1} \quad \text { for } i=1, \ldots, n-1
$$


define a nilpotent structure $\mu$ on $\mathfrak{n}$ so that $\left(\mathfrak{n}_{\mu}, Q\right)$ is nilsoliton. Writing the bracket relations with respect to the basis $X_{0}^{\prime}=X_{0}, X_{1}^{\prime}=X_{1}, X_{i}^{\prime}=$ $\left(\Pi_{i=1}^{i-1} a_{i}\right) X_{i}$ for $i=2$ to $n$ demonstrates that such a Lie algebra $\mathfrak{n}_{\mu}$ is isomorphic to $L_{n}$.

By Corollary 7, $\mathcal{B}$ is a Ricci eigenvector basis. The Gram matrix $U$ for $\left(\mathfrak{n}_{\mu}, Q\right)$ and $\mathcal{B}$ and the associated Cartan matrix $A=U-$ $[1]_{(n-1) \times(n-1)}$ are given by

$$
U=\left[\begin{array}{ccccc}
3 & 0 & 1 & \cdots & 1 \\
0 & 3 & 0 & \cdots & 1 \\
1 & 0 & 3 & \cdots & 1 \\
\vdots & \vdots & \vdots & \ddots & \vdots \\
1 & 1 & 1 & \cdots & 3
\end{array}\right] \quad \text { and } \quad A=\left[\begin{array}{ccccc}
2 & -1 & 0 & \cdots & 0 \\
-1 & 2 & -1 & \cdots & 0 \\
0 & -1 & 2 & \cdots & 0 \\
\vdots & \vdots & \vdots & \ddots & \vdots \\
0 & 0 & 0 & \cdots & 2
\end{array}\right]
$$

By Theorem 1, $\left(\mathfrak{n}_{\mu}, Q\right)$ is nilsoliton if and only if $v=\left[\alpha^{2}\right]$ is a solution of $U v=-2 \beta[1]_{(n-1) \times 1}$. Note that $A$ is the Cartan matrix of type $A_{n-1}$, so by Theorem 2 , the equation $U v=-2 \beta[1]_{(n-1) \times 1}$ has a unique positive solution for any $\beta<0$. By Theorem 2 , these solutions are in bijective correspondence with solutions to the systems $A v=\nu[1]_{(n-1) \times 1}$ for some $\nu>0$.

Writing out the equations of the system $A v=\nu[1]_{(n-1) \times 1}$, we find the recursion relation

$$
-v_{i-1}+2 v_{i}-v_{i+1}=\nu \quad\left(v_{0}=v_{n}:=0\right)
$$

which has solution $v_{i}=\frac{\nu}{2} i(n-i)$. The solutions $v_{i}$ are positive for all $i=1, \ldots, n-1$, so we may let

$$
a_{i}=\sqrt{v_{i}}=\sqrt{\frac{\nu}{2} i(n-i)}
$$

for $i=1, \ldots, n-1$ to define a nilsoliton metric Lie algebra $\left(\mathfrak{n}_{\mu}, Q\right)$. Note that $a_{i}=a_{n-i}$; this will be used in the proof of Theorem 35. Normalizing $\nu$ to be equal to two, and using the results of Example 11, we find that

$$
\begin{aligned}
\operatorname{ric}\left(X_{0}, X_{0}\right) & =-\frac{1}{2} \sum_{i=1}^{n-1} a_{i}^{2}-a_{i-1}^{2}=-\frac{1}{2}\left[n\left(\sum_{i=1}^{n-1} i\right)-\sum_{i=1}^{n-1} i^{2}\right] \\
& =-\frac{1}{12}(n-1) n(n+1), \\
\operatorname{ric}\left(X_{i}, X_{i}\right) & =-\frac{1}{2}(n+1)+i \text { for } 1 \leq i \leq n,
\end{aligned}
$$

and that the nilsoliton constant $\beta$ is $-\frac{1}{12}(n-1) n(n+1)-1$.

Uniqueness follows from the uniqueness of soliton metrics on nilpotent Lie groups ([Lau01a $]$ ).

q.e.d. 
9.3. Symmetric examples. In Theorem J of ([Heb98]), J. Heber showed that real and complex hyperbolic spaces are isolated among Einstein solvmanifolds and he predicted the existence of continuous families of Einstein solvmanifolds around quaternionic hyperbolic space $H^{m}(\mathbb{H})$ for $m \geq 2$ and around the Cayley plane. These families yield continuous families of soliton nilmanifolds around horospheres for the symmetric spaces. In this section we consider nilmanifolds associated to horospheres of rank one symmetric spaces of noncompact type, and we the find subfamilies of the families of soliton nilmanifolds associated to the Einstein solvmanifolds that Heber predicted. We also consider a horosphere for a higher rank symmetric space and find continuous families of soliton metrics around it.

First we consider the the Heisenberg algebra.

Example 26. Let $\mathfrak{h}_{n}$ be the $(2 n+1)$-dimensional Heisenberg algebra. The algebra $\mathfrak{h}_{n}$ may be represented with basis

$$
\mathcal{B}=\left\{X_{1}, \ldots, X_{n}, Y_{1}, \ldots, Y_{n}, Z\right\}
$$

and nontrivial structure constants coming from the bracket relations $\left[X_{i}, Y_{i}\right]=Z$ for $i=1, \ldots n$. We define the inner product $Q$ on $\mathfrak{h}_{n}$ so that $\mathcal{B}$ is orthonormal. It is a Ricci eigenvector basis by Corollary 7.

The root vectors for $\left(\mathfrak{h}_{n}, Q\right)$ with respect to $\mathcal{B}$ are linearly independent, and the $n \times n$ Gram matrix $U$ has $u_{i i}=3$ for $i=1, \ldots, n$ and $u_{i j}=1$ for $i \neq j$. The graph $S(U)$ is the complete graph on $n$ vertices, and if $A=U-[1]_{n \times n}$, the graph $S(A)$ is $n$ vertices with no edges. The graph is regular so a constant weighting assigning the value of one to each vertex satisfies Equation (2), and hence $\left(\mathfrak{h}_{n}, Q\right)$ is a nilsoliton metric Lie algebra. Because $A=U-[1]_{(2 n+1) \times(2 n+1)}=2 \mathrm{Id}$ is nonsingular, by Theorem 2 , the weighting is unique. No matter what sign choice we make $( \pm 1)$ for the structure constants, we get an algebra isomorphic to $\mathfrak{h}_{n}$. Thus, $\left(\mathfrak{h}_{n}, Q\right)$ is the only nilsoliton metric Lie algebra inside its equivalence class $\Omega_{\Lambda}$.

The nilmanifold corresponding to the metric Lie algebra $\left(\mathfrak{h}_{n}, Q\right)$ in the previous example is isometric to any horosphere for complex hyperbolic space of real dimension $2 n+2$, a rank one symmetric space of noncompact type. In the next example, we consider a horosphere for quaternionic symmetric space. C. Gordon and M. Kerr presented other continuous families around quaternionic hyperbolic spaces in GK01] and in [Ker06].

Example 27. Let $\mathfrak{v}=\mathbb{R}^{8}$ and $\mathfrak{z}=\mathbb{R}^{3}$, and let

$$
\mathcal{B}=\left\{V_{1}, i V_{1}, j V_{1}, k V_{1}, V_{2}, i V_{2}, j V_{2}, k V_{2}, i Z, j Z, k Z\right\}
$$


be a orthonormal basis for the vector space $\mathfrak{n}=\mathfrak{v} \oplus \mathfrak{z}$ in the obvious way. Let $\mu$ be an element of $\Lambda^{2} \mathfrak{n}^{*} \otimes \mathfrak{n}$ with nontrivial structure constants coming from the twelve bracket relations

$$
\begin{aligned}
{\left[V_{s}, i V_{s}\right] } & =\alpha_{1 i}^{i}(s) i Z & & {\left[j V_{s}, k V_{s}\right]=\alpha_{j k}^{i}(s) i Z } \\
{\left[V_{s}, j V_{s}\right] } & =\alpha_{1 j}^{j}(s) j Z & {\left[i V_{s}, k V_{s}\right] } & =-\alpha_{i k}^{j}(s) j Z \\
{\left[V_{s}, k V_{s}\right] } & =\alpha_{1 k}^{k}(s) k Z & {\left[i V_{s}, j V_{s}\right] } & =\alpha_{i j}^{k}(s) k Z
\end{aligned}
$$

$(s=1,2)$. By Corollary [7, $\mathcal{B}$ is a Ricci eigenvector basis for all possible values of these structure constants. After writing out the matrices $U$ and $A$ and using Maple to solve $U v=[1]_{12 \times 1}$, we find a four parameter family of solutions for squares of structure coefficients.

$$
\begin{aligned}
& \alpha_{1 i}^{i}(1)^{2}=\alpha_{j k}^{i}(1)^{2}=\frac{1}{10}+s+t \\
& \alpha_{1 j}^{j}(1)^{2}=\alpha_{i k}^{j}(1)^{2}=\frac{1}{10}-s \\
& \alpha_{1 k}^{k}(1)^{2}=\alpha_{i j}^{k}(1)^{2}=\frac{1}{10}-t \\
& \alpha_{1 i}^{i}(2)^{2}=\alpha_{j k}^{i}(2)^{2}=\frac{1}{10}-(s+t) \\
& \alpha_{1 j}^{j}(2)^{2}=\alpha_{i k}^{j}(2)^{2}=\frac{1}{10}+s \\
& \alpha_{1 k}^{k}(2)^{2}=\alpha_{i j}^{k}(2)^{2}=\frac{1}{10}+t
\end{aligned}
$$

Notice that $\mathfrak{n}_{\mu}$ is always two-step, so the Jacobi identity holds trivially for any $\mu$ defined by any choice of structure constants.

It can be checked that the graph $S(U)$ has as its symmetry group the dihedral group $D_{6}$; therefore the solution space shares this symmetry. The Lie algebra structure for the horosphere of quaternionic hyperbolic space of dimension twelve comes from setting all parameter values equal to zero. Solutions are positive when $|s|,|t|$ and $|s+t|$ are less than $1 / 10$. It may be checked that the eigenvalues for Ric are $-3 / 20$ on $\mathfrak{v}$ and $4 / 20$ on $\mathfrak{z}$ and that $\beta=-1 / 2$, so $D$ has eigenvalues $7 / 20$ and $14 / 20$ on $\mathfrak{v}$ and $\mathfrak{z}$ respectively. The Ricci endomorphism and $D$ are the same for all the nilsoliton Lie algebras in $\Omega_{\Lambda}$, so the Einstein solvable extensions all have the same Ricci endomorphism.

By considering the values trace $J_{W}^{2}$, for unit $W \in \mathfrak{z}$, it is easy to see that varying $s$ and $t$ so that $0 \leq s \leq t \leq 1 / 10$ and $s+t \leq 1 / 10$, defines a family of metric Lie algebras that are not pairwise isometrically isomorphic; hence, by Wil82] the corresponding nilmanifolds are not isometric. Thus we have found a two-parameter family of soliton metric Lie algebras.

J. Heber showed that there exists an 84-parameter family of Einstein solvmanifolds around the Cayley plane with the symmetric metric. Here we analyze the system $U v=[1]$ that would lead to members 
THE EXISTENCE OF SOLITON METRICS FOR NILPOTENT LIE GROUPS 37

of that family having same set of nonzero structure constants relative to a natural basis.

Example 28. Let $\mathfrak{v}=\mathbb{R}^{8}$ and $\mathfrak{z}=\mathbb{R}^{7}$, and let

$$
\mathcal{B}=\left\{V_{0}, V_{1}, V_{2}, V_{3}, V_{4}, V_{5}, V_{6}, V_{7}, Z_{1}, Z_{2}, Z_{3}, Z_{4}, Z_{5}, Z_{6}, Z_{7}\right\}
$$

be an orthonormal basis for $\mathfrak{n}=\mathfrak{v} \oplus \mathfrak{z}$ in the obvious way. Consider a Lie bracket $\mu$ with nontrivial structure constants coming from the bracket relations

$$
\left[V_{i}, V_{j}\right]=\alpha_{i j}^{k} Z_{k}
$$

where $(i, j, k)$ ranges over the set

$$
\begin{aligned}
& \{(0,1,1),(2,4,1),(3,7,1),(5,6,1),(0,2,2),(1,4,2),(3,5,2), \\
& (6,7,2),(0,3,3),(1,7,3),(2,5,3),(4,6,3),(0,4,4),(1,2,4), \\
& (3,6,4),(5,7,4),(0,5,5),(1,6,5),(2,3,5),(4,7,5),(0,6,6), \\
& \quad(1,5,6),(2,7,6),(3,4,6),(0,7,7),(1,3,7),(2,6,7),(4,5,7)\}
\end{aligned}
$$

By Corollary $7, \mathcal{B}$ is a Ricci eigenvector basis for all metric Lie algebra structures of this form. When all the structure constants are equal, $\mathfrak{n}_{\mu}$ is the Lie algebra of $N$ from the Iwasawa decomposition $K A N$ of the isometry group of the Cayley plane. After finding the root vectors, computing the Gram matrix $U$, and using MAPLE to solve 
$U\left[\alpha^{2}\right]=[1]_{28 \times 1}$ we get an eight-dimensional set of solutions

$$
\begin{aligned}
& \left(\alpha_{01}^{1}\right)^{2}=\frac{1}{18}+a+b+c+d+2 e \\
& \left(\alpha_{24}^{1}\right)^{2}=\frac{1}{18}+a+b-c-d-2 e \\
\left(\alpha_{37}^{1}\right)^{2}= & \left(\alpha_{56}^{1}\right)^{2}=\frac{1}{18}-a-b \\
& \left(\alpha_{02}^{2}\right)^{2}=\frac{1}{18}-a-2 e+f+h \\
& \left(\alpha_{14}^{2}\right)^{2}=\frac{1}{18}-a+2 e-f-h \\
\left(\alpha_{35}^{2}\right)^{2}= & \left(\alpha_{67}^{2}\right)^{2}=\frac{1}{18}+a \\
& \left(\alpha_{03}^{3}\right)^{2}=\frac{1}{18}-c-e-f+g \\
& \left(\alpha_{17}^{3}\right)^{2}=\frac{1}{18}-d-e+f-\frac{1}{2} g+h \\
\left(\alpha_{25}^{3}\right)^{2}= & \left(\alpha_{46}^{3}\right)^{2}=\frac{1}{18}+\frac{1}{2} c+\frac{1}{2} d+e-\frac{1}{2} g-\frac{1}{2} h \\
& \left(\alpha_{04}^{4}\right)^{2}=\frac{1}{18}-b+\frac{1}{2} c-\frac{1}{2} d-2 e+f+\frac{1}{2} g+\frac{1}{2} h \\
& \left(\alpha_{12}^{4}\right)^{2}=\frac{1}{18}-b-\frac{1}{2} c+\frac{1}{2} d+2 e-f-\frac{1}{2} g-\frac{1}{2} h \\
\left(\alpha_{36}^{4}\right)^{2}= & \left(\alpha_{57}^{4}\right)^{2}=\frac{1}{18}+b \\
& \left(\alpha_{05}^{5}\right)^{2}=\frac{1}{18}-\frac{1}{2} c-\frac{1}{2} d+2 e+\frac{1}{2} g-\frac{1}{2} h \\
& \left(\alpha_{16}^{5}\right)^{2}=\frac{1}{18}-\frac{1}{2} c-\frac{1}{2} d-2 e-\frac{1}{2} g+\frac{1}{2} h \\
& \left(\alpha_{23}^{5}\right)^{2}=\frac{1}{18}+c \\
& \left(\alpha_{47}^{5}\right)^{2}=\frac{1}{18}+d \\
& \left(\alpha_{06}^{6}\right)^{2}=\frac{1}{18}+e \\
& \left(\alpha_{15}^{6}\right)^{2}=\frac{1}{18}-3 e \\
\left(\alpha_{27}^{6}\right)^{2}= & \left(\alpha_{34}^{6}\right)^{2}=\frac{1}{18}+e \\
& \left(\alpha_{07}^{7}\right)^{2}=\frac{1}{18}-f-g-h \\
& \left(\alpha_{13}^{7}\right)^{2}=\frac{1}{18}+f \\
& \left(\alpha_{26}^{7}\right)^{2}=\frac{1}{18}+g \\
& \left(\alpha_{45}^{7}\right)^{2}=\frac{1}{18}+h \\
& \\
& \\
&
\end{aligned}
$$

To see that the metric nilpotent Lie algebras in the family defined by these solutions are not all isomorphic, assume there is an isometric isomorphism $\phi$ between two elements $(\mathfrak{n}, Q)$ and $\left(\mathfrak{n}^{\prime}, Q^{\prime}\right)$ of the family. The map $\phi$ must preserve the center $\mathfrak{z}=\operatorname{span}\left\{Z_{i}\right\}_{i=1}^{7}$ and the orthogonal complement of the center $\mathfrak{v}=\operatorname{span}\left\{V_{i}\right\}_{i=0}^{7}$. The natural grading automorphism that is the identity on $\mathfrak{v}$ and twice the identity on $\mathfrak{z}$ defines gradings of $\mathfrak{n}$ and $\mathfrak{n}^{\prime}$. We use this grading to apply Theorem 9 
The set $S$ of values $K(X \wedge Y)=-\frac{3}{4}\left\|\operatorname{ad}_{X} Y\right\|^{2}$ for unit $X, Y \in \mathfrak{v}$, is the same for $\left(\mathfrak{n}_{1}, Q_{1}\right)$ and $\left(\mathfrak{n}_{2}, Q_{2}\right)$. Therefore, the set $S$ is an isometry invariant.

Clearly the set $S$, modulo rescaling, takes on infinitely many values as the structure constants vary over the set of solutions we have found, so there is continuous family of soliton metrics on nilpotent Lie algebras, pairwise nonhomothetic, defined by the solutions given above.

In the next example, we consider a horosphere of maximal dimension for the rank $n-1$ symmetric space $\mathcal{P}_{n}=S L_{n}(\mathbb{R}) / S O(n)$. Let $\mathfrak{t}_{n}$ denote the vector space of strictly upper triangular $n \times n$ matrices. We use $\mathcal{B}=\left\{E_{i j} \mid 1 \leq i<j \leq n\right\}$ as a basis, where $E_{i j}$ is the matrix with a one in the $(i, j)$ position and zeroes elsewhere, and we let $Q$ be the inner product such that $\mathcal{B}$ is orthonormal. Let $\alpha_{i j k}$ denote the structure constant $\left\langle\left[E_{i j}, E_{j k}\right], E_{i k}\right\rangle$, for $1 \leq i<j<k \leq n$. The nonzero structure constants are indexed by $\Theta_{n}$. Let $\mu_{0}$ be the element of $\Lambda^{2} \mathfrak{t}_{n}^{*} \otimes \mathfrak{t}_{n}$ with $\alpha_{i j k}=1$ for all $(i, j, k)$ in $\Theta_{n}$; the nilmanifold corresponding to the metric Lie algebra $\left(\left(\mathfrak{t}_{n}\right)_{\mu_{0}}, Q\right)$ is isometric to a horosphere for a regular geodesic in the symmetric space $\mathcal{P}_{n}$.

Theorem 29. If $n \leq 4$, then $\left(\left(\mathfrak{t}_{n}\right)_{\mu_{0}}, Q\right)$ is the only nilsoliton in its equivalence class $\Omega_{\Lambda}$ up to scaling. For $n>4$, there are continuous families of nonisometric nilsoliton metrics around $\left(\left(\mathfrak{t}_{n}\right)_{\mu_{0}}, Q\right)$ in its equivalence class $\Omega_{\Lambda}$.

Proof. The dimension of $\mathfrak{t}_{n}$ is $\left(\begin{array}{c}n \\ 2\end{array}\right)$, and there are $\left(\begin{array}{c}n \\ 3\end{array}\right)$ nonzero structure constants $\alpha_{i j k}$ (where $(i, j, k)$ is in $\Theta_{n}$ ). It is easily checked that the Jacobi equation holds for all choices of structure constants, noting that the only nonzero triple brackets of basis vectors are of the form $\left[E_{i j},\left[E_{j k}, E_{k l}\right]\right]$, where $(i, j, k)$ is in $\Theta_{5}$.

If $n=3$, then $\left(\left(\mathfrak{t}_{3}\right)_{\mu_{0}}, Q\right)$ is the three-dimensional Heisenberg Lie algebra. If if $n=4$, a brief computation shows that $\left(\left(\mathfrak{t}_{4}\right)_{\mu_{0}}, Q\right)$ is the unique nilsoliton metric (up to scaling) in its equivalence class $\Omega_{\Lambda}$.

When $n=5$, the dimension of $\mathfrak{t}_{5}$ is $\left(\begin{array}{l}5 \\ 2\end{array}\right)=10$ and $\|\Lambda\|=\left(\begin{array}{l}5 \\ 3\end{array}\right)$. also ten. There exists a continuous family of nilsoliton metrics around $\left(\left(\mathfrak{t}_{5}\right)_{\mu_{0}}, Q\right)$. MAPLE shows that the system $U v=[1]_{10 \times 1}$ has general 
solution

$$
\left[\begin{array}{l}
\left(\alpha_{123}\right)^{2} \\
\left(\alpha_{124}\right)^{2} \\
\left(\alpha_{125}\right)^{2} \\
\left(\alpha_{234}\right)^{2} \\
\left(\alpha_{235}\right)^{2} \\
\left(\alpha_{134}\right)^{2} \\
\left(\alpha_{345}\right)^{2} \\
\left(\alpha_{145}\right)^{2} \\
\left(\alpha_{245}\right)^{2} \\
\left(\alpha_{135}\right)^{2}
\end{array}\right]=\frac{1}{5}\left[\begin{array}{l}
1 \\
1 \\
1 \\
1 \\
1 \\
1 \\
1 \\
1 \\
1 \\
1
\end{array}\right]+s\left[\begin{array}{c}
1 \\
-1 \\
0 \\
-1 \\
0 \\
1 \\
0 \\
0 \\
0 \\
0
\end{array}\right]+t\left[\begin{array}{c}
0 \\
1 \\
-1 \\
1 \\
-1 \\
0 \\
-1 \\
1 \\
0 \\
0
\end{array}\right]+u\left[\begin{array}{c}
0 \\
0 \\
0 \\
1 \\
-1 \\
0 \\
-1 \\
0 \\
1 \\
0
\end{array}\right]+v\left[\begin{array}{c}
1 \\
0 \\
-1 \\
0 \\
-1 \\
0 \\
0 \\
0 \\
0 \\
1
\end{array}\right] .
$$

By Theorem 3, the Ricci endomorphisms are all the same for all choices of structure constants, so we need to use sectional curvature to distinguish nonisometric examples. To show that $\left(\mathfrak{t}_{\mu}, Q\right)$ for $\mu$ where $(s, t, u, v) \neq(0,0,0,0)$ are not isometric to $\left(\left(\mathfrak{t}_{5}\right)_{\mu_{0}}, Q\right)$, one can argue as in Example 28, applying Theorem 9 using the canonical grading of $\mathfrak{t}_{5}$ as the sum $\bigoplus_{k=1}^{4} \mathfrak{g}_{k}$, where $\mathfrak{g}_{k}=\operatorname{span}_{\mathbb{R}}\left\{E_{i j} \in \mathcal{B}: k=j-i\right\}$, for $k=1, \ldots, 4$.

If $n>5$, then $\left(\begin{array}{c}n \\ 2\end{array}\right)-\left(\begin{array}{c}n \\ 3\end{array}\right)>2$, so $U$ is singular by Corollary 23. The standard $\mu_{0}$ gives a positive solution to the nilsoliton linear system, so by continuity, there is a continuous family of positive solutions around the symmetric solution in its class $\Omega_{\Lambda}$. As in the $n=5$ case, the sectional curvature serves as an invariant to distinguish the perturbed metric Lie algebras from the standard one.

q.e.d.

9.4. Regular graphs. Recall that a graph is regular if its automorphism group acts transitively on vertices. With respect to natural choices of bases, the nilsoliton metric Lie algebras defined by horospheres of rank one symmetric spaces of noncompact type have generalized Dynkin diagrams that are regular graphs. The structure vectors are of the form [1], defining weightings that assign the value one to each vertex of the generalized Dynkin diagram. The regularity of the graph insures that Equation (2) holds for some $\beta$, so that [1] is a solution of $U v=-2 \beta[1]$. In general, we have the following corollary to Theorem 1 .

Corollary 30. Let $(\mathfrak{n}, Q)$ be an inner product space with Ricci eigenvector basis $\mathcal{B}$. If the generalized Dynkin diagram $S(U)$ associated to a nontrivial level set $\Omega_{\Lambda}$ in $\Lambda^{2} \mathfrak{n}^{*} \otimes \mathfrak{n}$ is a regular graph, then any $\mu$ in $\Omega_{\Lambda}$ with structure vector [1] is a nilsoliton metric.

Observe that [1] is a solution to $U v=-2 \beta[1]$ if and only if the columns of $U$ all sum to $-2 \beta$. 
A large class of examples of nilsoliton metric Lie algebras with regular generalized Dynkin diagrams was presented by E. DeLoff [Del79]. A two-step metric nilpotent Lie algebra $(\mathfrak{n}, Q)$ is called uniform of type $(m, n, r)$ if there exists an orthonormal basis

$$
\mathcal{B}=\left\{X_{1}, \ldots, X_{n}, Z_{1}, \ldots Z_{m}\right\}
$$

and numbers $r$ and $s$ such that

(1) $\left[V_{i}, V_{j}\right] \in\left\{0, \pm Z_{1}, \ldots, Z_{m}\right\},\left[V_{i}, Z_{l}\right]=\left[Z_{k}, Z_{l}\right]=0$,

(2) If $\left[V_{i}, V_{j}\right]= \pm\left[V_{i}, V_{k}\right]$, then $V_{j}=V_{k}$

(3) For every $Z_{l}$ there exist exactly $r$ disjoint pairs $\left\{V_{i}, V_{j}\right\}$ with $\left[V_{i}, V_{j}\right]=Z_{l}$

(4) For every $V_{i}$ there exist exactly $s V_{j}$ with $\left[V_{i}, V_{j}\right] \neq 0$.

Notice that the number of nonzero bracket relations is $s n=2 \mathrm{rm}$.

Example 31. ([Del79], see also [Wol91]) A uniform metric Lie algebra is always nilsoliton. Properties (1) and (2) imply that $\left\langle\operatorname{ad}_{X_{i}}, \operatorname{ad}_{X_{j}}\right\rangle=0$ for $i \neq j$ and $\left\langle J_{Z_{k}}, J_{Z_{l}}\right\rangle=0$ for $k \neq l$. The basis $\mathcal{B}$ is then a Ricci eigenvector basis by Corollary [7. Property 3 implies that $\left\|J_{Z_{l}}\right\|=2 r$ for all $l$ and Property 4 makes $\left\|\operatorname{ad}_{X_{i}}\right\|=s$ for all $i$ The generalized Dynkin diagram $S(U)$ is a regular graph with $s n$ vertices and $2 s+r$ edges at each vertex, so by Corollary $[30,(\mathfrak{n}, Q)$ is nilsoliton. Then Theorem 8 gives that Ricci eigenvalues $\kappa_{X_{i}}=-\frac{1}{2} s$ and $\kappa_{Z_{l}}=\frac{1}{2} r$. By Theorem 16, the nilsoliton constant is $\beta=-\frac{1}{2}(2 s+r)$. By Corollary 23, if the number of nonzero structure constants $s n=2 \mathrm{rm}$ is larger than the dimension $m+n$ then there are continuous families of solutions to $U v=[1]$. Example 27 is uniform of type $(m, n, r)=(3,8,4)$ and Example 28 is uniform of type $(m, n, r)=(7,8,4)$; in both cases $\|\Lambda\|=$ $r m>m+n=\operatorname{dim} \mathfrak{n}$ and there are continuous families of nilsolitons around the solitons from symmetric spaces.

The next example shows that admitting a positive definite symmetric derivation is not a sufficient condition for nilpotent Lie algebra to admit a nilsoliton metric.

Theorem 32. There exists a filiform nilpotent Lie algebra that admits a positive definite derivation but does not admit a nilsoliton metric.

Proof. Let $\mathfrak{n}$ be the seven-dimensional Lie algebra with basis $\mathcal{B}=$ $\left\{X_{i}\right\}_{i=1}^{7}$ with bracket relations defined by

$$
\begin{array}{ll}
{\left[X_{1}, X_{i}\right]=X_{i+1}} & \text { for } i=2, \ldots, 6 \\
{\left[X_{2}, X_{i}\right]=X_{i+2}} & \text { for } i=3,4,5
\end{array}
$$

This is the algebra $R_{6}$ from the infinite family of filiform Lie algebras $R_{n}$ described in [Kha89]. The endomorphism $A$ defined by $A\left(X_{i}\right)=i X_{i}$ 
for $i=1$ to 7 is a positive definite derivation of $\mathfrak{n}$, and it is the only one up to scaling (See [GH94] and Theorem 2.12 of [Kha89]).

The proof is by contradiction. Suppose that $Q$ is an inner product on $\mathfrak{n}$ so that $(\mathfrak{n}, Q)$ is a nilsoliton metric Lie algebra with nilsoliton constant $\beta$. Eigenvectors for $D$ are also eigenvalues for $\operatorname{Ric}=D+\beta \operatorname{Id}$. The positive definite derivation $D=\operatorname{Ric}-\beta \mathrm{Id}$ is a multiple of $A$ so the vectors in the $\mathcal{B}$ must be eigenvectors for the derivation.

As the multiplicities of the eigenvalues of $A$ are all one, we may rescale each basis vector in $\mathcal{B}$ and order the basis that we have a Ricci eigenvector basis $\mathcal{B}^{\prime}=\left\{X_{i}^{\prime}=a_{i} X_{i}\right\}_{i=1}^{7}$ where $A\left(X_{i}\right)=i X_{i}$ for $i=$ $1, \ldots, 7$. The structure vector $\left[\alpha^{2}\right]$ depends on the values of $a_{1}, \ldots, a_{7}$, but the set

$$
\Lambda(\mathfrak{n}, \mathcal{B})=\{(1, i, i+1)\}_{i=2}^{6} \cup\{(2, i, i+2)\}_{i=3}^{5}
$$

does not, as $a_{i} \neq 0$ for all $i=1, \ldots 7$. By Theorem $1,-\frac{1}{2}\left[\alpha^{2}\right]$ is a positive solution to $U v=[1]_{7 \times 1}$. Using MAPLE, we find the general solution to $U v=[1]_{7 \times 1}$ is

$$
\begin{aligned}
v & =\frac{1}{5}(0,-1,0,3,2,3,0,0)^{T}+s v_{1}+t v_{2}, \quad \text { where } \\
v_{1} & =(0,1,0,-1,0,-1,1,0)^{T} \text { and } \\
v_{2} & =(0,1,1,-1,-1,-1,0,1)^{T} \text { and } s, t \in \mathbb{R} .
\end{aligned}
$$

But the first component of the general solution is always zero, a contradiction. Thus, $\mathfrak{n}$ does not admit a nilsoliton metric. q.e.d.

We conclude the section with an example of a Einstein solvmanifold of rank two found using the ideas of this section.

Example 33. Let $\Lambda=\{(1,2,3),(1,3,4),(1,4,5),(2,3,5)\}$. This is the set $\Lambda(\mathfrak{n}, \mathcal{B})$ for one of the filiform metric Lie algebras $L_{n}$ described in Subsection 9.2. We saw in Theorem 25] that letting $\alpha_{12}^{3}=\alpha_{14}^{5}=\sqrt{3}$ and $\alpha_{13}^{4}=2$ defines a nilsoliton metric Lie algebra with $\beta=-11 / 2$ and Ricci vector $\mathbf{R i c}=-\frac{1}{2}(-10,-3,-1,1,3)^{T}$. Then eigenvalue vector of the derivation $D_{1}=\mathrm{Ric}-\beta \mathrm{Id}$ with respect to $\mathcal{B}$ is

$$
v_{D_{1}}=\mathbf{R i c}+\frac{11}{2}[1]_{5 \times 1}=\frac{1}{2}(2,9,11,13,15)^{T} .
$$

Letting $\left[A_{1}, X_{i}\right]=D_{1}\left(X_{i}\right)$ defines a rank one Einstein solvmanifold $\left(\mathfrak{s}_{1}=\left\langle A_{1}\right\rangle \oplus \mathfrak{n}, \widetilde{Q}_{1}\right)$.

Now notice that $\Lambda$ is a subset of $\Sigma_{5}$ and the generalized Dynkin diagram $S(U)$ is a minor graph of type $A_{2} \oplus A_{1}$ in $S\left(\Sigma_{5}\right)$, which is of type $A_{3}$. Hence $\left(L_{5}, Q\right)$ admits another symmetric derivation with eigenvalues $1,2,3,4,5$ each of multiplicity one. The commuting symmetric derivations encoded by $\boldsymbol{v}_{1}=(2,9,11,13,15)^{T}$ and $\boldsymbol{v}_{2}=(1,2,3,4,5)^{T}$ 
are in the derivation algebra of $\mathfrak{n}$. Using Gram-Schmidt orthogonalization, we find a vector $\boldsymbol{v}_{2}^{\prime}=(26,-33,-7,19,45)^{T}$ orthogonal to $\boldsymbol{v}_{1}$ so that $\operatorname{span}\left\{\boldsymbol{v}_{1}, \boldsymbol{v}_{2}^{\prime}\right\}=\operatorname{span}\left\{\boldsymbol{v}_{1}, \boldsymbol{v}_{2}\right\}$. Let $D_{2}$ be the derivation defined by the vector $\boldsymbol{v}_{2}^{\prime}$. Finally, define the metric solvable extension $\left(\mathfrak{s}_{2}=\left\langle A_{1}, A_{2}\right\rangle \oplus \mathfrak{n}, \widetilde{Q}\right)$ by taking $\left\{A_{1}, A_{2}\right\} \cup \mathcal{B}$ as an orthonormal basis and setting $\operatorname{ad}_{A_{1}}=D_{1}$ and $\operatorname{ad}_{A_{2}}=D_{2}$.

It can be checked, using formulas for the Ricci curvature of Einstein solvmanifolds of Iwasawa type in [Wo191] that $\left(\mathfrak{s}_{2}, Q\right)$ is an Einstein solvmanifold of rank two with nonpositive sectional curvature.

In Heb98, Heber asked which types of eigenvalue vectors $v_{D}=$ $\left(\mu_{1}, \ldots, \mu_{n}\right)$ occur for the derivations $D=$ Ric $-\beta$ Id defining rank one Einstein extensions of nilsolitons. We remark that the question can be rephrased as follows. The Ricci vector for a nilsoliton $(\mathfrak{n}, Q)$ and Ricci eigenvector basis $\mathcal{B}$ is of the form Ric $=v_{D}+\beta[1]_{n \times 1}$. On the other hand, by Theorem 8 , Ric $=-\frac{1}{2} Y^{T}\left[\alpha^{2}\right]$, where $\left[\alpha^{2}\right]$ is the structure vector for $(\mathfrak{n}, Q)$ with respect to $\mathcal{B}$. Hence, $-\frac{1}{2} Y^{T}\left[\alpha^{2}\right]=$ $\left(\mu_{1}, \ldots, \mu_{n}\right)^{T}+\beta[1]_{n \times 1}$. Thus, in order to prove that there is a nilsoliton $(\mathfrak{n}, Q)$ of eigenvalue type $v_{D}$, we would need need to show that the vector $-2\left(v_{D}+\beta[1]_{n \times 1}\right)$ is in the closure of the positive cone spanned by root vectors in $\left\{Y_{i j}^{k} \mid(i, j, k) \in \Sigma_{\left(\mu_{1}, \ldots, \mu_{n}\right)}\right\}$. Notice that $v_{D}$ itself is orthogonal to the cone.

\section{AdDing NiLsolitons}

Now we prove Theorem 4 .

Proof. When $\Lambda\left(\mathfrak{n}_{\mu_{1}}, \mathcal{B}\right)$ and $\Lambda\left(\mathfrak{n}_{\mu_{1}}, \mathcal{B}\right)$ are disjoint sets, it can be seen from the expression for the Ricci form in Theorem 6 that the nilRicci endomorphism is linear with respect to the Lie bracket defining it. Since $\mathcal{B}$ is a Ricci eigenvector basis for $\left(\mathfrak{n}_{\mu_{1}}, Q\right)$ and $\left(\mathfrak{n}_{\mu_{2}}, Q\right)$, this linearity implies that $\mathcal{B}$ is also a Ricci eigenvector basis for the sum $\left(\mathfrak{n}_{\mu_{1}+\mu_{2}}, Q\right)$, and the Ricci vector for $\mathfrak{n}_{\mu_{1}+\mu_{2}}$ with respect to $\mathcal{B}$ is

$$
\operatorname{Ric}_{\mu_{1}+\mu_{2}}^{\mathcal{B}}=\operatorname{Ric}_{\mu_{1}}^{\mathcal{B}}+\operatorname{Ric}_{\mu_{2}}^{\mathcal{B}} \text {. }
$$

Now we consider the quantities $Y_{i j}^{k} \mathbf{R i c}_{\mu_{1}+\mu_{2}}$ as $(i, j, k)$ varies over $\Lambda\left(\mathfrak{n}_{\mu_{1}+\mu_{2}}, \mathcal{B}\right)$. If the triple $(i, j, k)$ is in the set $\Lambda\left(\mathfrak{n}_{\mu_{1}}, \mathcal{B}\right)$, then

$$
\begin{aligned}
Y_{i j}^{k} \mathbf{R i c}_{\mu_{1}+\mu_{2}} & =Y_{i j}^{k}\left(\mathbf{R i c}_{\mu_{1}}+\mathbf{R i c}_{\mu_{2}}\right) \\
& =Y_{i j}^{k} \mathbf{R i c}_{\mu_{1}}+Y_{i j}^{k} \mathbf{R i c}_{\mu_{2}} \\
& =-2 \beta_{1}+c_{2}
\end{aligned}
$$

and similarly, if $(i, j, k)$ is in $\Lambda\left(\mathfrak{n}_{\mu_{2}}, \mathcal{B}\right)$, then $Y_{i j}^{k} \mathbf{R i c}_{\mu_{1}+\mu_{2}}=-2 \beta_{2}+c_{1}$. If $-2 \beta_{1}+c_{2}=-2 \beta_{2}+c_{1}$, the quantity $Y_{i j}^{k} \mathbf{R i c}_{\mu_{1}+\mu_{2}}$ is constant as $Y_{i j}^{k}$ 
varies over $\Lambda\left(\mathfrak{n}_{\mu_{1}+\mu_{2}}, \mathcal{B}\right)$. By Theorem 16, the metric algebra $\left(\mathfrak{n}_{\mu_{1}+\mu_{2}}, Q\right)$ is a nilsoliton with nilsoliton constant $\beta=-2 \beta_{1}+c_{2}$.

q.e.d.

A trivial application of Theorem 4 is that the direct sum of nilsoliton metric Lie algebras with the same nilsoliton constant is a nilsoliton metric Lie algebra. More generally, one can think of defining a new metric nilpotent Lie algebra from an old one by adding new basis vectors and new bracket relations; sometimes Theorem 4 can be used to show that such a procedure defines a new nilsoliton metric Lie algebra, as we illustrate in the following example.

Example 34. Let $(\mathfrak{n}, Q)$ be a nilsoliton metric Lie algebra with nilsoliton constant $\beta$ and Ricci eigenvector basis $\mathcal{B}$. Let $Z$ be an element of the Ricci eigenvector basis in the center of $\mathfrak{n}$ with positive eigenvalue $\kappa_{Z}$. (Such an element exists because the Ricci endomorphism is positive definite on the center by Theorem 6.) Let $\mathfrak{n}^{\prime}=\mathbb{R}^{2} \oplus \mathfrak{n}$. Fix basis vectors $A$ and $B$ in $\mathbb{R}^{2}$ and endow $\mathfrak{n}^{\prime}$ with the metric which restricts to $Q$ on $\mathfrak{n}$ and makes $A$ and $B$ orthonormal to to each other and orthogonal to $\mathfrak{n}$. Define a nilpotent structure on $\mathfrak{n}^{\prime}$ by adding bracket relation $[A, B]=a Z$ to the bracket relations already defined by the nilpotent structure on $\mathfrak{n}$. The Jacobi identity holds for $\mathfrak{n}^{\prime}$, and $\mathfrak{n}^{\prime}$ is nilpotent. If $a^{2}=\frac{-4 \beta-2 \kappa_{Z}}{5}$, then Theorem 4 can be used to show that $\left(\mathfrak{n}^{\prime}, Q^{\prime}\right)$ is a nilsoliton metric Lie algebra. The Heisenberg algebra of dimension $2 n+3$ may be constructed from the Heisenberg algebra of dimension $2 n+1$ using this method.

For odd $n \geq 3$, the $n=(2 m+1)$-dimensional filiform nilpotent Lie algebra $Q_{n}$ is defined relative to the basis $\left\{X_{i}\right\}_{i=0}^{n}$ by

$$
\begin{aligned}
{\left[X_{0}, X_{i}\right] } & =X_{i+1}, \quad i=1, \ldots, n-2 \\
{\left[X_{i}, X_{n-i}\right] } & =(-1)^{i} X_{n}, \quad i=1, \ldots, n-1 .
\end{aligned}
$$

Theorem 35. For all $n \geq 3$, the Lie algebra $Q_{n}$ admits a unique nilsoliton metric.

Proof. Let $Q$ be the inner product on $\mathfrak{n}$ with respect to which $\mathcal{B}$ is orthonormal. By Corollary $7, \mathcal{B}$ is a Ricci eigenvector basis, even if we change the values of the nonzero structure constants.

The set of nonzero structure constants coming from brackets defined in Equation (13) is

$$
\Lambda_{1}=\{(0,1,2),(0,2,3), \ldots,(0, n-3, n-1)\}
$$

and the set of nonzero structure constants coming from brackets defined in Equation (14) is

$$
\Lambda_{2}=\{(1, n-1, n),(2, n-2, n), \ldots,(m, m+1, n)\} .
$$


Notice that $n$ does not occur in any triple in $\Lambda_{1}$, and that 0 does not occur in any triple in $\Lambda_{2}$, and the two sets of triples are disjoint.

The bracket relations in Equation (13) are the same as those for the nilpotent Lie algebra $L_{n-1}$, as in Theorem 25. In the proof of that theorem, we saw that letting $\left[X_{0}, X_{i}\right]=\sqrt{i(n-1-i)} X_{i+1}$ defines a nilsoliton metric algebra $\left(\mathfrak{n}_{\mu_{1}}, Q\right)$ isomorphic to $L_{n-1} \oplus \mathbb{R}$ with Ricci vector

$\operatorname{Ric}_{\mu_{1}}^{\mathcal{B}}=\left(-\frac{1}{12}(n-2)(n-1) n,-\frac{1}{2}(n-2),-\frac{1}{2}(n-2)+1, \ldots, \frac{1}{2}(n-2), 0\right)^{T}$ and nilsoliton constant $\beta_{1}=-\frac{1}{12}(n-2)(n-1) n-1$.

The root vectors $Y_{i j}^{k}$ for bracket relations in Equation (14) are the same as those for the $(2 m+1)$-dimensional Heisenberg algebra $\mathfrak{h}_{m}$ as presented in Example 26. Letting $\alpha_{i j}^{k}=1$ for all $(i, j, k) \in \Lambda_{2}$ defines a nilsoliton metric Lie algebra $\left(\mathfrak{n}_{\mu_{2}}, Q\right)$ isomorphic to $\mathbb{R} \oplus \mathfrak{h}_{m}$ with Ricci vector

$$
\operatorname{Ric}_{\mu_{2}}^{\mathcal{B}}=-\frac{1}{2}(0,1,1, \ldots, 1,-m)^{T}
$$

and nilsoliton constant $\beta_{2}=-\frac{1}{2}(2+m)$.

It can be checked that for all $Y_{i j}^{k}$ with $(i, j, k)$ in the second index set $\Lambda_{2}$, the product $Y_{i j}^{k} \mathbf{R i c}_{\mu_{1}}^{\mathcal{B}}$ is zero, and for all $Y_{i j}^{k}$ with $(i, j, k)$ in the first index set $\Lambda_{1}$, the product $Y_{i j}^{k} \mathbf{R i c}_{\mu_{2}}^{\mathcal{B}}$ is zero.

If we rescale the structure constants for $\mathfrak{n}_{\mu_{1}}$ and $\mathfrak{n}_{\mu_{2}}$ by $\sqrt{-\beta_{2}}$ and $\sqrt{-\beta_{1}}$ respectively, then the rescaled metric nilpotent Lie algebras $\mathfrak{n}_{-\beta_{2} \mu_{1}}$ and $\mathfrak{n}_{-\beta_{1} \mu_{2}}$ both have nilsoliton constant $-\beta_{1} \beta_{2}$. By Theorem 10, the metric algebra $\left(\mathfrak{n}=\mathfrak{n}_{\sqrt{-\beta_{2}}} \mu_{1}+\sqrt{-\beta_{1}} \mu_{2}, Q\right)$ satisfies the nilsoliton condition with nilsoliton constant $-\beta_{1} \beta_{2}$.

Using the change of basis

$$
\begin{aligned}
X_{0}^{\prime} & =\frac{1}{\sqrt{-\beta_{2}}} X_{0} \\
X_{1}^{\prime} & =X_{1} \\
X_{i}^{\prime} & =\left(\Pi_{i=1}^{i-1} a_{i}\right) X_{i} \quad \text { for } i=2 \text { to } n-1 \\
X_{n}^{\prime} & =\sqrt{-\beta_{1}} X_{n}
\end{aligned}
$$

and the symmetry $a_{i}=a_{n-1-i}$, it can be shown that $\mathfrak{n}$ is isomorphic to $Q_{n}$.

Thus, we have shown that $\left(\mathfrak{n}_{-\beta_{2} \mu_{1}-\beta_{1} \mu_{2}}, Q\right)$ is a nilsoliton metric Lie algebra isomorphic to $Q_{n}$. Uniqueness follows from [Lau01a]. q.e.d.

Given a $k$-step nilpotent Lie algebra $\mathfrak{n}$, a graded Lie algebra gr $\mathfrak{n}$ is defined by on the vector space gr $\mathfrak{n}=\bigoplus_{i=1}^{k}\left(\mathfrak{n}^{(i-1)} / \mathfrak{n}^{(i)}\right)$ by the bracket structure

$$
\left[x+\mathfrak{n}^{(i)}, y+\mathfrak{n}^{(j)}\right]=[x, y]+\mathfrak{n}^{(i+j)}, \quad x \in \mathfrak{n}^{(i-1)}, y \in \mathfrak{n}^{(j-1)} .
$$


If $\mathfrak{n}$ is isomorphic to gr $\mathfrak{n}$, then $\mathfrak{n}$ is called naturally graded. In Ver70, M. Vergne showed that every naturally graded filiform Lie algebra is isomorphic to $L_{n}$ or $Q_{n}$. So in Theorems 25] and 35] we have shown:

Corollary 36. Every naturally graded filiform Lie algebra admits a Ricci nilsoliton metric that is unique up to scaling.

\section{ACKNOWLEDGments}

The author is grateful to Jorge Lauret, Gerald Payne and Wayne Polyzou for helpful comments, and to Dennis Stowe for valuable discussions.

\section{REFERENCES}

[Ale75] D. V. Alekseevskiǔ, Homogeneous Riemannian spaces of negative curvature, Mat. Sb. (N.S.) 96(138) (1975), 93-117, 168. MR 50 \#14587

[Bes87] Arthur L. Besse, Einstein manifolds, Ergebnisse der Mathematik und ihrer Grenzgebiete (3) [Results in Mathematics and Related Areas (3)], vol. 10, Springer-Verlag, Berlin, 1987. MR 88f:53087

[CK04] Bennett Chow and Dan Knopf, The Ricci flow: an introduction, Mathematical Surveys and Monographs, vol. 110, American Mathematical Society, Providence, RI, 2004. MR MR2061425

[Del79] E. Deloff, Naturally reductive metrics and metrics with volume preserving geodesic symmetries, thesis, Rutgers, 1979.

[DL57] J. Dixmier and W. G. Lister, Derivations of nilpotent Lie algebras, Proc. Amer. Math. Soc. 8 (1957), 155-158. MR 18,659a

[DM82] Isabel Dotti Miatello, Ricci curvature of left invariant metrics on solvable unimodular Lie groups, Math. Z. 180 (1982), no. 2, 257-263. MR 84a:53044

[Ebe04] Patrick Eberlein, Geometry of 2-step nilpotent Lie groups, Modern dynamical systems and applications, Cambridge Univ. Press, Cambridge, 2004, pp. 67-101. MR MR2090766

[Fav72] Gabriel Favre, Une algèbre de Lie caractéristiquement nilpotente de dimension 7, C. R. Acad. Sci. Paris Sér. A-B 274 (1972), A1338-A1339. MR 45 \#3495

[GH94] Michel Goze and You Hakimjanov, Sur les algèbres de Lie nilpotentes admettant un tore de dérivations, Manuscripta Math. 84 (1994), no. 2, 115-124. MR MR1285951 (95g:17011)

[GK01] Carolyn S. Gordon and Megan M. Kerr, New homogeneous Einstein metrics of negative Ricci curvature, Ann. Global Anal. Geom. 19 (2001), no. 1, 75-101. MR 2002f:53067

[Ham82] Richard S. Hamilton, Three-manifolds with positive Ricci curvature, J. Differential Geom. 17 (1982), no. 2, 255-306. MR MR664497 (84a:53050)

[Heb98] Jens Heber, Noncompact homogeneous Einstein spaces, Invent. Math. 133 (1998), no. 2, 279-352. MR 99d:53046

[HJ90] Roger A. Horn and Charles R. Johnson, Matrix analysis, Cambridge University Press, Cambridge, 1990, Corrected reprint of the 1985 original. MR 91i:15001 
THE EXISTENCE OF SOLITON METRICS FOR NILPOTENT LIE GROUPS 47

[Jen69] Gary R. Jensen, Homogeneous Einstein spaces of dimension four, J. Differential Geometry 3 (1969), 309-349. MR MR0261487 (41 \#6100)

[Kac90] Victor G. Kac, Infinite-dimensional Lie algebras, third ed., Cambridge University Press, Cambridge, 1990. MR 92k:17038

[Kar93] Ron Karidi, Ricci structure and volume growth for left invariant Riemannian metrics on nilpotent and some solvable Lie groups, Geom. Dedicata 46 (1993), no. 3, 249-277. MR 94e:53048

[Ker06] Megan M. Kerr, A deformation of quaternionic hyperbolic space, Proc. Amer. Math. Soc. 134 (2006), no. 2, 559-569 (electronic). MR MR2176025 (2007e:53050)

[Kha89] Yu. B. Khakimdzhanov, Characteristically nilpotent Lie algebras, Algebra i Logika 28 (1989), no. 6, 722-737, 744. MR 92a:17016

[Kha02] Yusupdjan Khakimdjanov, Characteristically nilpotent, filiform and affine Lie algebras, Recent advances in Lie theory (Vigo, 2000), Res. Exp. Math., vol. 25, Heldermann, Lemgo, 2002, pp. 271-287. MR 2003m:17011

[Lau] Jorge Lauret, Einstein solvmanifolds are standard, arXiv:math.DG/0703472v1.

[Lau01a] Jorge Lauret, Ricci soliton homogeneous nilmanifolds, Math. Ann. 319 (2001), no. 4, 715-733. MR 2002k:53083

[Lau01b] _ Standard Einstein solvmanifolds as critical points, Q. J. Math. 52 (2001), no. 4, 463-470. MR 2002j:53048

[Lau02] _ Finding Einstein solvmanifolds by a variational method, Math. Z. 241 (2002), no. 1, 83-99. MR 2003g:53064

$[\mathrm{McD}] \quad$ Carlan McDaniel, Generalized Dynkin diagrams for low-dimensional nilsoliton Lie algebras, undergraduate research project directed by Tracy Payne.

[Pera] Grisha Perelman, The entropy formula for the Ricci flow and its geometric applications, arXiv:mathDG/0211159.

[Perb] - Finite extinction time for the solutions to the Ricci flow on certain three-manifolds, arXiv:mathDG/0307245.

[Perc] Ricci flow with surgery on three-manifolds, arXiv: mathDG/0303109.

[Ver70] Michèle Vergne, Cohomologie des algèbres de Lie nilpotentes. Application à l'étude de la variété des algèbres de Lie nilpotentes, Bull. Soc. Math. France 98 (1970), 81-116. MR 44 \#6797

[Wil82] Edward N. Wilson, Isometry groups on homogeneous nilmanifolds, Geom. Dedicata 12 (1982), no. 3, 337-346. MR MR661539 (84a:53048)

[Wil03] Cynthia Will, Rank-one Einstein solvmanifolds of dimension 7, Differential Geom. Appl. 19 (2003), no. 3, 307-318. MR 2013098

[Wol91] T. H. Wolter, Einstein metrics on solvable groups, Math. Z. 206 (1991), no. 3, 457-471. MR 92b:53074

Department of Mathematics, Idaho State University, Pocatello, ID 83209-8085

E-mail address: payntrac@isu.edu 